\title{
Mammary ductal epithelium controls cold-induced adipocyte thermogenesis
}

\section{Luis Santos}

Icahn School of Medicine at Mount Sinai

Douglas Arneson

University of California

Alexandra Alvarsson

Icahn School of Medicine at Mount Sinai

Karthickeyan Krishnan

University of California

Alessia Centzone

Université Libre de Bruxelles

Sanil Patel

Icahn School of Medicine at Mount Sinai

Shani Sadeh

Icahn School of Medicine at Mount Sinai https://orcid.org/0000-0002-6892-7043

In Sook Ahn

University of California

Graciel Diamante

University of California

Ingrid Cely

University of California

Atul Butte

Bakar Institute for Computational Health Sciences, University of California, San Francisco https://orcid.org/0000-0002-7433-2740

\section{Cédric Blanpain}

Université Libre de Bruxelles https://orcid.org/0000-0002-4028-4322

\section{Sarah Stanley}

Icahn School of Medicine at Mount Sinai

Aldons Lusis

University of California Los Angeles https://orcid.org/0000-0001-9013-0228

\section{Xia Yang}

University of California

Prashant Rajbhandari ( $\nabla$ prashant.rajbhandari@mssm.edu )

Icahn School of Medicine at Mount Sinai https://orcid.org/0000-0002-8146-3861 


\section{Biological Sciences - Article}

Keywords:

Posted Date: February 1st, 2022

DOl: https://doi.org/10.21203/rs.3.rs-1179254/v1

License: (c) (i) This work is licensed under a Creative Commons Attribution 4.0 International License. Read Full License 
2

3

4

Luis C. Santos ${ }^{1,8}$, Douglas Arneson ${ }^{2,5,8}$, Alexandra Alvarsson ${ }^{1,8}$, Karthickeyan Chella Krishnan ${ }^{3}$, Alessia Centzone ${ }^{4}$, Sanil Patel ${ }^{1}$, Shani Sadeh ${ }^{1}$, In Sook Ahn ${ }^{5}$, Graciel Diamante ${ }^{5}$, Ingrid Cely ${ }^{5}$, Atul J. Butte $2,6,7$, Cédric Blanpain ${ }^{5}$, Sarah A. Stanley ${ }^{1}$, Aldons J. Lusis ${ }^{3}$, Xia Yang ${ }^{5}$, and Prashant Rajbhandari ${ }^{1,9}$

${ }^{1}$ Diabetes, Obesity, and Metabolism Institute, Icahn School of Medicine at Mount Sinai, New York, NY USA

${ }^{2}$ Bakar Computational Health Sciences Institute, University of California, San Francisco, CA USA ${ }^{3}$ Department of Medicine/Division of Cardiology and Department of Human Genetics, University of California, Los Angeles, CA USA

${ }^{4}$ Laboratory of Stem Cells and Cancer, Université Libre de Bruxelles (ULB), Brussels, Belgium

${ }^{5}$ Department of Integrative Biology and Physiology and Bioinformatics Interdepartmental Program, University of California, Los Angeles, CA USA

${ }^{6}$ Department of Pediatrics, University of California, San Francisco, CA, USA

${ }^{7}$ Center for Data-Driven Insights and Innovation, University of California Health, Oakland, CA, USA

${ }^{8}$ Equal Contribution

${ }^{9}$ Corresponding author:

Prashant Rajbhandari PhD

Diabetes, Obesity, and Metabolism Institute

Department of Endocrinology and Bone Disease

Icahn School of Medicine at Mount Sinai

1 Gustave L. Levy Place

New York, NY 10029 USA

Office (212) 659-8653

E-mail: prashant.rajbhandari@mssm.edu

Main Figures: 4; Extended Data Figures: 4; Supplemental Movies: 2 


\section{ABSTRACT}

38 Sympathetic activation during cold exposure increases adipocyte thermogenesis via expression of

39 mitochondrial protein uncoupling protein $1(\mathrm{UCP} 1)^{1}$. The propensity of adipocytes to express

40 UCP1 is under a critical influence of the adipose microenvironment and varies among various fat

$41 \operatorname{depots}^{2-7}$. Here we report that cold-induced adipocyte UCP1 expression in female mouse

42 subcutaneous white adipose tissue ( $\mathrm{scWAT}$ ) is regulated by mammary gland ductal epithelial cells

43 in the adipose niche. Single cell RNA-sequencing (scRNA-seq) show that under cold condition

44 glandular alveolar and hormone-sensing luminal epithelium subtypes express transcripts that

45 encode secretory factors involved in regulating adipocyte UCP1 expression. We term mammary

46 duct secretory factors as "mammokines". Using whole-tissue immunofluorescence 3D

47 visualization, we reveal previously undescribed sympathetic nerve-ductal points of contact and

48 show that sympathetic nerve-activated mammary ducts limit adipocyte UCP1 expression via cold-

49 induced mammokine production. Both in vivo and ex vivo ablation of mammary ductal epithelium

50 enhances cold-induced scWAT adipocyte thermogenic gene program. The mammary duct network

51 extends throughout most scWATs in female mice, which under cold exposure show markedly less

52 UCP1 expression, fat oxidation, energy expenditure, and subcutaneous fat mass loss compared to

53 male mice. These results show a previously uncharacterized role of sympathetic nerve-activated

54 glandular epithelium in adipocyte thermogenesis. Overall, our findings suggest an evolutionary

55 role of mammary duct luminal cells in defending glandular adiposity during cold exposure,

56 highlight mammary gland epithelium as a highly active metabolic cell type, and implicate a

57 broader role of mammokines in mammary gland physiology and systemic metabolism. 
61 The scWAT depots in female mice are mostly mammary gland WATs (mgWAT) which is highly

62 heterogenous tissue consisting of adipocytes, preadipocytes, mesenchymal stem cells, immune

63 cells, endothelial cells, SNS nerve fibers, and mammary epithelial cells forming a ductal structure.

64 The epithelial cells are divided into myoepithelial/basal cells, and luminal cells, which can be

65 luminal hormone sensing, or not ${ }^{8}$. In virgin female mice, the mammary gland already has ductal

66 structures in the anterior and posterior scWAT and metabolic cooperativity between luminal ductal

67 cells and stroma is known to be important for mammary gland function and development ${ }^{8,9}$.

68 Profound changes in mammary ducts and adipocytes are seen during gestation, pregnancy,

69 lactation, and post-involution ${ }^{10,11}$. The importance of adipocytes for mammary duct

70 morphogenesis, the dedifferentiation of adipocytes during lactation, and reappearance during

71 lactation post-involution, all suggest a dynamic homeostatic interplay between ductal luminal

72 epithelial cells and adipocytes ${ }^{11-13}$. It is not clear, however, what paracrine signaling programs

73 from mammary ducts regulate adipocyte metabolism and thermogenesis. Notably, our current-

74 state-of knowledge of WAT thermogenesis and UCP1 expression is mostly based on male

75 scWATs, which lacks mammary glandular epithelial cells. Importantly, besides the role of

76 immune cells in the adipose microenvironment, the contribution of other cell types in controlling

77 adipocyte UCP1 expression is still not clear.

78 To study cellular heterogeneity, inter-tissue communication, and cellular transcription

79 dynamics in mgWAT in a thermogenic condition, we isolated the stromal vascular fraction (SVF)

80 from the mgWAT of 10 -week-old virgin female mice exposed to 24 -hour cold $\left(\mathrm{COLD}, 4^{\circ} \mathrm{C}\right)$ or

81 room temperature (RT) and performed scRNA-seq (Fig. 1A). We obtained 12,222 cells and used

82 Cell Ranger software from $10 \mathrm{X}$ Genomics for data processing and the $\mathrm{R}$ package Seurat ${ }^{14}$ to 
83 generate cell clusters and resolve their identities as previously described ${ }^{15}$ (see Methods). We

84 integrated our dataset with eight other publicly available single cell datasets, from mammary gland

85 tissues including Tabula Muris and Tabula Muris Senis ${ }^{16-19}$ : i) for cell type identification, ii) to

86 increase confidence in the projected cell type, iii) for sex and age differences, and iv) for mammary

87 gland development (Extended Data Fig. 1A, and Table 1). This integrated dataset allowed us to

88 precisely annotate various cell types present in mgWAT of female mice. Further subclustering of

89 our integrated dataset based on known cell type marker genes identified clusters of a) adipocyte

90 precursor cells (APCs), b) B cells, c) macrophages, d) T cells, e) endothelial cells, f) immune

91 precursor cells (IPC), g) dendritic cells (DC) , h) Schwann cells, i) myoepithelial cells (myoep),

92 and j) luminal-hormone sensing (Luminal-HS), luminal-alveolar (Luminal-AV), luminal-HS-AV,

93 and myoepithelial cells (Fig. 1B, Extended Data Fig. 1B and 1C).

94 To gain insight into the remodeling of stromal cells under adrenergic stress, we segregated

95 the cumulative tSNE-plot into RT and COLD treatment by animal replicate. The tSNE and dot 96 plots reveal global changes in relative proportions of SVF clusters between RT and COLD (Fig.

97 1C). Among all the clusters luminal cells showed significant differences in cell type percentages

98 (RT 1:7.2\%, RT 2: 7.5\%; COLD 1: 16.9\%, COLD 2: 19.1\%) and appeared to have large

99 differences in their global transcriptomic profiles in the t-SNE two-dimensional projection where

100 cells from RT and COLD were segregated (Fig. 1C). To quantitatively determine the

101 transcriptional impact of cold treatment on individual cell types, we characterized differentially

102 expressed genes as a function of cluster types and found a high degree of transcriptional variation

103 in luminal HS and AV under the cold conditions (Extended Data Fig. 1D). Further subclustering

104 of luminal epithelial cell types (luminal HS, luminal AV, and luminal HS-AV) revealed marked

105 differences in clustering at RT and COLD ${ }^{20}$ (Fig. 1C and Extended Data Fig. 1D, 1E). 
Luminal cell clusters showed remarkable transcriptional differences in cell clusters

107 between RT and cold, implicating a potential remodeling of the luminal epithelium upon cold

108 exposure (Fig. 1C, 1D and Extended Data Fig. 1D-1F). To probe for factors that are differentially

109 expressed in luminal cells under cold exposure, we performed differential gene expression (DEG)

110 analysis on RT and cold exposed luminal subclusters. We found upregulation of Wnt4, Adropin

111 (Enho), leucine rich alpha-2 glycoprotein ( $\operatorname{rg} 1)$, Diglyceride acyltransferase (Dgat2), haptoglobin

112 (Hp), and angiopoietin-like 4 (Angptl4) in Luminal HS cells, lipocalin-2 (Lcn2), Angptl4, and

113 Apolipoprotein B editing complex (Apobec3) in Luminal AV cells, and Lrg1, neuregulin 4 (Nrg4),

114 ceruloplasmin (Cp), Angptl4 in Luminal HS-AV cells (Fig. 1E-1G, Extended Data Fig. 1G). Many

115 of these genes (shown by red arrows in Fig. 1E-1G) encode secreted factors that play important

116 roles in local and systemic lipid metabolism ${ }^{21-30}$. t-SNE plots of normalized gene expression levels

117 for cold-induced mammokines in mgWAT (our study), male scWAT SVFs and mature

118 adipocytes ${ }^{31}$ show that Angptl4 is also expressed by most mature adipocytes; however, other cold-

119 induced mammokine genes showed relatively localized expression in ductal epithelial cell

120 adhesion molecule (Epcam)+ cells (Extended Data Fig. 1G and 1H). Our RNAscope fluorescent

121 in situ hybridization (FISH) analysis showed a highly localized expression of mammokines Enho,

122 Mfge8, Lrg1, Lcn2, Hp, Nrg4, and Wnt4 in Epcam+ and Krt8+ (luminal ductal epithelial markers)

123 in mammary ductal luminal cells (Fig. 1H).

124 Shifts in the luminal epithelium transcriptomic state with cold and localized expression of

125 beta-adrenergic receptors, $A d r b 2$ and $A d r b 1$ expression in luminal cells, suggests that these cells 126 may directly respond to cold-induced SNS activation ${ }^{32-34}$ (Fig. 1C and Extended Data Fig. 1G). To

127 determine if duct epithelial cells are innervated by sympathetic nerves, we used Adipoclear, a 128 robust protocol based on immunolabeling-enabled three-dimensional imaging of solvent-cleared 
129 organ iDISCO (22), for high-resolution, three-dimensional imaging of mammary tissue. We 130 analyzed the 3D distribution and density of a sympathetic marker, tyrosine hydroxylase (TH), and

131 its relationship to EPCAM+ mammary ductal cells in mgWAT from mice exposed to RT or cold.

132 Cold exposure caused prominent morphological changes in mammary ducts such as increased 133 EPCAM+ branching and terminal ductal bifurcations (Fig. 2A and Extended Data Fig. 2A), which 134 is consistent with data showing increased branch morphogenesis upon isoproterenol treatment ${ }^{35}$. 135 Our data further reveal that nerve fibres are interwoven with mammary gland ducts and alveolar 136 structures in mgWATs (Supplementary Movie 1). However, we did not see an effect of cold 137 treatment on duct volume, nerve volume, or the ratio of duct-to-nerve volume (Fig. 2B). To 138 examine interactions between sympathetic innervation and mammary gland ducts in more detail, 139 we performed confocal imaging in six regions of the mgWAT fat pad from each of 6 RT and 6 140 cold-treated mice. Consistent with our scRNA-seq data, we saw a significant increase in EPCAM 141 staining in the ducts of cold-exposed mgWAT (Fig 2C and 2D). We also identified contacts 142 between $\mathrm{TH}+$ fibres and EPCAM+ ducts (neuroductal points) with a trend to increased volume of 143 nerve contacts (normalized for duct volume) in cold-treated mice ( $p=0.09$, Fig S2F). Interestingly, 144 TH intensity, which has been reported to increase with sympathetic activation, was significantly 145 higher at the neuroductal points in cold exposed mgWAT compared to controls (Fig 2C, 2E, 2F 146 Supplementary Movie 2). Similarly, EPCAM intensity was also significantly elevated at 147 neuroductal points in keeping with local induction of expression. However, there were no 148 significant changes in duct or nerve volume or in $\mathrm{TH}$ intensity across the whole nerve volume 149 (Extended Data Fig 2C-2H). Taken together, our data show a significant remodelling of mammary 150 ducts and their contacts with sympathetic innervation upon cold exposure. 
To explore the biological relevance of cold-induced increase in i) luminal subtype 152 population transcriptional state (Fig. 1) and ii) EPCAM intensity and SNS innervation of 153 mammary ducts (Fig. 2), we first purified EPCAM+ and EPCAM- cells from mgWAT of RT and 154 cold-exposed mice and then stained for EPCAM and CD49F for fluorescence-activated cell sorting 155 (FACS) analysis to probe for luminal cell population changes under cold stress. As shown in 156 Extended Data Fig. 3A, we noticed three distinct populations of cells that were EPCAM ${ }^{\text {lo }} \mathrm{CD} 49 \mathrm{~F}^{\mathrm{lo}}$ 157 (stromal), EPCAM ${ }^{\text {hi }}$ CD49F ${ }^{\text {hi }}$ (luminal), and EPCAM $^{\text {lo }} \mathrm{CD} 49 \mathrm{~F}^{\text {hi }}$ (basal) cells. Luminal cells were 158 only enriched in EPCAM+ purified cells and showed a cold-dependent increase in cell population 159 compared to RT conditions. We also noticed a marked reduction in the basal cell population upon 160 cold treatment in EPCAM+ selected cells, consistent with the scRNA-seq data in Fig. 1C 161 (myoepithelial cluster, RT: 2.15\%, COLD: 0.65\%). These data indicate a differential response to 162 cold stress by luminal and basal cells in ducal epithelium (Extended Data Fig. 3A). To test whether 163 luminal cells directly responded to SNS activation, we tested mammokine expression in isolated 164 primary EPCAM+ mgWAT that were either treated or not treated with isoproterenol. Cold-induced 165 mammokines showed increased expression upon isoproterenol (beta-adrenergic receptor agonist) 166 treatment (Extended Data Fig. 3B). To determine whether adrenergic-activated luminal cells are 167 involved in mgWAT adipose thermogenesis, we depleted EPCAM+ cells from mgWAT SVFs by 168 positive selection using magnetic cell sorting (MACS) and differentiated SVFs ex vivo with and 169 without ductal cells into beige adipocytes (Fig. 3A). Depletion of EPCAM+ cells from SVFs of 170 RT mgWAT potentiated expression of thermogenic genes such as Ucp1, Cox $8 b$, Ppargcla and 171 Cidea and this potentiation of thermogenic genes was markedly amplified in the cold-exposed 172 condition (Fig. 3A and Extended Data Fig 3C). To further test the crosstalk of epithelial cells and 173 adipocytes, we developed an in vitro co-culture system involving a controlled mixture of 
174 adipogenic 10T1/2 cells with nontransformed mouse mammary gland (NMuMG cells) (derived 175 from "normal" mammary epithelium). Seeding density of as low as 2.5\% NMuMG cells resulted 176 in a significant reduction of $U c p 1$ and beiging potential of $10 \mathrm{~T} 1 / 2$ cells compared to the pure 177 culture (Fig. 3B and Extended Data Fig. 3D-3F). The higher the fraction of NMuMG cells in the 178 co-culture, the lower the relative expression of $U c p 1$ and other thermogenic genes such as $\operatorname{Cox} 8 B$ 179 and Ppargcla measured by RT-qPCR (Fig. 3B). To test the ex vivo and in vivo role of ductal 180 epithelial cells in adipose thermogenesis, we compared aged-matched female mgWAT with three 181 complimentary ductal ablated models; i) estrogen receptor-alpha (ERa) knockout mice (Esrl KO), 182 ii) male mice which lack or possess only rudimentary glandular ducts, iii) inguinal (ducts) or 183 dorsolumbar (no ducts) portion of mgWAT from 5-week-old female mice. First, to test the role of 184 ductal cells in adipose thermogenesis, we isolated SVFs from male iWATs and female mgWATs 185 and differentiated them into beige adipocytes in the presence or absence of isoproterenol. We 186 found that SVFs from male iWATs show markedly higher beiging and isoproterenol-mediated $187 U c p 1$ expression compared to EPCAM+ SVFs from female mgWAT (Fig. 3C and Extended Data 188 Fig. 3G). In agreement with the ex vivo data, female mgWATs showed significantly less 189 expression of cold-induced thermogenic genes such as Ucpl, Cox $8 b$, and Ppargcla compared to 190 male iWATs (Fig. 3D). Since mgWATs make up almost all of the subcutaneous fat mass in female 191 mice, we reasoned that highly reduced adipocyte thermogenic gene expression could potentially 192 influence whole body energy metabolism. To test this, we performed indirect calorimetry on age193 matched male and female mice at RT and $24 \mathrm{hr}$ cold exposure using a metabolic chamber. We 194 found that female mice showed highly reduced energy expenditure (EE), oxygen consumption 195 (VO2), and carbon dioxide production (VCO2) during cold exposure compared to male mice (Fig. $1963 \mathrm{E}$ and Extended Data Fig. 3I). Female mice showed markedly higher respiratory exchange ratio 
197 (RER) than males under cold exposure, indicating a decrease in cold-induced fat oxidation and the 198 possibility that mgWATs maintain adiposity under cold stress (Fig. 3E and Extended Data Fig. 199 3J). Generalized linear model (GLM)-based regression analyses showed a significant group and 200 interaction effect in RER between males and females based on fat mass as a covariate (Extended 201 Data Fig. 3J). We did not see significant differences in locomotor activities and food consumption 202 between the sexes (Extended Data Fig. 3K). These RER data were further supported by our 203 magnetic resonance imaging (MRI) body composition analysis which showed that male mice lose 204 significant body weight and fat mass during cold stress whereas females show no differences 205 before and after cold stress (Fig.3F and Extended Data Fig. 3H). Next, we compared the cold206 induced thermogenic gene expression program between WT and Estrogen Receptor-alpha (Esr1 207 KO) mice. Esrl KO mice are known to possess hypoplastic mammary ducts and remain 208 rudimentary throughout the life span of a female mouse ${ }^{36}$. As shown in Fig. 3G, cold-exposed Esr 1

209 KO mice showed markedly increased expression of $U c p 1$, and other thermogenic genes compared 210 to WT control. In the 5 weeks after birth, mammary ducts are concentrated in the inguinal portion 211 closer to the nipple and are confined near the lymph node and virtually absent toward the 212 dorsolumbar region of the mgWAT, providing distinct anatomical regions within the mgWAT to 213 test the role of ductal epithelium in adipose thermogenesis ${ }^{37}$. 5-week-old female mice were 214 exposed to cold and inguinal and dorsolumbar regions were dissected to assess thermogenic 215 transcripts. Epcam transcripts were present only in the inguinal region, however, thermogenic 216 genes were mostly similar between inguinal and dorsolumbar region except for Ucpl where we 217 saw an increase in $U c p 1$ levels in inguinal part (Fig. 3H). Chi et. al reported regional differences 218 between inguinal and dorsolumbar region and there could be a regional control of $U c p 1$ expression 219 in 5-week-old mice independent of ductal cells ${ }^{38}$. However, consistent with our data, we noticed 
220 significantly less expression of genes involved in lipid mobilization such as Pnpla2 and Hsl, in

221 inguinal regions compared to dorsolumbar, indicating that the ductal epithelium is potentially

222 inhibiting cold-induced lipid mobilization. All these data point, for the first time, toward a possible

223 unique mechanism of SNS-activated mgWAT under cold stress to limit thermogenesis and 224 preserve adiposity.

225 Our results show a unique possible SNS-mediated crosstalk between mammary ductal cells 226 and adipocytes to control adipocyte thermogenesis. Our differentially expressed genes in 227 mammary ductal cells under adrenergic stimulation showed upregulation of genes that encode 228 factors such as Angpt14, Enho, Lrg1, and Lcn2, which are known to play inhibitory roles in 229 adipocyte thermogenesis. These factors also showed high enrichment in mammary Epcam + cells 230 by scRNA-seq and RNAscope in situ hybridization. Among these factors, Lcn2 was previously 231 shown to inversely correlate with $U c p 1$ expression in female gonadal WAT (gWAT) ${ }^{24}$. Female 232 gWATs, which lack mammary ducts, express exceedingly high levels of Ucpl compared to male $233 \mathrm{gWATs}^{24}$. Reciprocally, we find that cold-stressed female mgWATs express high levels of Lcn2 234 and low levels of Ucp 1 compared to males (Fig. 3D and Extended Data Fig. 4A). The secretion of 235 Lcn2 by luminal AV and HS-AV cells could potentially be a mechanism of luminal cells to block 236 excess thermogenesis and preserve adiposity. Consistent with data in Fig.1 and Fig.3, both 237 isoproterenol treatment and cold exposure led to an increase in Lcn2 levels in Epcam + mammary 238 ducts (Extended Data Fig. 4B). To test adrenergic-dependent expression of Lcn2 protein 239 expression in luminal cells, we used organoids from a genetic mouse model that is based on 240 doxycycline (Dox)-based tet-responsive diphtheria toxin A (DTA) system derived from 241 interbreeding two transgenic strains: (1) mice expressing the tetracycline-on ("tet-on') 242 transcription factor rtTA under the control of the luminal epithelial cell-specific Krt8 gene 
243 promoter (K8rtTA); (2) mice expressing tet-responsive DTA (TetO-Cre) that can be activated in

244 the presence of Dox. This model enables luminal cell ablation in a temporally regulated manner.

245 Mammary duct organoids isolated from K8rtTA-DTA mice ${ }^{39}$ show increases in Lcn2 protein

246 expression upon isoproterenol treatment and these increases were diminished upon Dox treatment

247 (Fig. 4A and Extended Fig. 4C-4H), showing a direct response of luminal cells to produce Lcn2.

248 To test the physiological role of Lcn2 in regulating mgWAT thermogenesis, we mimicked cold

249 induction of Lcn2 production in mgWAT by inducing Len2 exogenous expression specifically in

250 mgWAT of $L c n 2 \mathrm{KO}$ mice by injecting adipoAAV-Lcn2 or adipoAAV-GFP24 (See Methods). Our

251 unbiased bulk RNA-seq data from the mgWATs of adipoAAV-Lcn2 injected mice show that Lcn2

252 expression was not supraphysiological compared to controls (Fig. 4B). The volcano plot in Fig.

253 4B demonstrates that Lcn2 exogenous expression significantly decreased the expression of

254 thermogenic genes such as Ucp1, Cidea, Ppara and increased expression of adipogenic genes

255 including Lep, Mmp12 $2^{40,41}, Z f p 423^{42}$, and Lbp ${ }^{43}$. Lcn2 overexpression also led to an increase in

256 Aldhlal, which was recently shown to inhibit adipose thermogenesis by downregulating UCP1

257 levels ${ }^{44,45}$. We validated our RNA-seq data by directed qPCR in both Len2 reconstituted mgWAT

258 of Lcn2 KO and WT mice (Fig. 4C) and treated beige differentiated SVFs isolated from Lcn2 KO

259 mgWATs with and without recombinant Len2 (Extended Data Fig. 4I). Finally, to test the role of

260 Lcn2 under cold stress, female Lcn2 $\mathrm{KO}$ and WT mice were exposed to cold $\left(4^{\circ} \mathrm{C}\right)$ for $24 \mathrm{hr}$.

261 Compared to controls, cold-exposed Lcn2 KO mice mgWAT showed more beiging/browning and

262 gene expression analysis showed a significant increase in thermogenic genes such as Ucpl

263 indicating that $\operatorname{Lcn} 2$ is potentially one of the limiting factors involved in regulating the propensity

264 of mgWAT to beige (Extended Data Fig. 4J). Overall, our scRNA-seq analysis and both our tissue-

265 specific gain-of-function and loss-of-function experimental data show Lcn2 as a factor expressed 
266 in luminal cells that could function to inhibit thermogenesis and maintain adiposity in mgWAT

267 during cold-stress.

268 In summary, our studies have uncovered a direct inhibitory role of mammary duct luminal

269 cells in adipocyte $U c p 1$ expression and thermogenic gene program. SNS fibers directly innervate

270 EPCAM+ luminal cells and adrenergic stimulation of luminal cells transduce expression of

271 mammokines such LCN2 that regulates Ucp1 expression in mgWAT adipocytes under cold

272 exposure. Depletion of EPCAM+ luminal epithelial cells potentiate the capacity of ex vivo

273 differentiated mgWAT preadipocytes to express UCP1, and female mice with ductal epithelium

274 loss show higher cold-induced UCP1 expression compared to controls. Importantly, female mice

275 demonstrate significantly less scWAT adipocyte UCP1 expression compared to male mice under

276 cold exposure. Taken together, these findings provide a new insight into mammary gland biology,

277 expand our understanding of the role of adipose microenvironment in adipocyte UCP1 expression,

278 and reveal the potential of mammokines to regulate local and systemic energy homeostasis.

279

280 METHODS

281 Animal Studies

282 C57BL/6 WT male and female mice (\#000664), ESR1 KO (\#004744), LCN2KO (\#24630) were

283 acquired from Jackson Laboratory and maintained in a pathogen-free barrier-protected

284 environment $\left(12: 12 \mathrm{~h} \mathrm{light} / \mathrm{dark}\right.$ cycle, $\left.22^{\circ} \mathrm{C}-24^{\circ} \mathrm{C}\right)$ at the UCLA and Mount Sinai animal facilities.

285 The Krt8rtTA-TetO-DTA mouse model was described previously ${ }^{39}$. For the time course cold 286 exposure experiment, WT mice at $8-10$ weeks of age were singly housed at $4^{\circ} \mathrm{C}$ room in a non-

287 bedded cage without food and water for first $6 \mathrm{~h}$; thereafter food, water, and one cotton square 288 were added. For the $24 \mathrm{~h}$ harvest, $3 \mathrm{~h}$ before harvest, food, water, and cotton square were removed 
and then mice were harvested. At the end of the experiment, mgWATs were resected for analysis.

290 For overexpression studies, recombinant adeno-associated virus serotype 8 (AAV8) expressing

291 LCN2 or GFP was generated and injected as described previously ${ }^{24}$. Indirect calorimetry was

292 performed using Promethion Systems (Sable Systems, Las Vegas, NV). Animals were placed

293 individually in chambers at ambient temperature $\left(22.0^{\circ} \mathrm{C}\right)$ for $21 \mathrm{hr}$ followed by $24 \mathrm{hr}$ cold $(4.0$

$294{ }^{\circ} \mathrm{C}$ ) with $12 \mathrm{hr}$ light/dark cycles. Animals had free access to food and water. Respiratory

295 measurements were made in 5 min intervals after initial 7-9 hr acclimation period. Energy

296 expenditure was calculated from VO2 and RER using the Lusk equation, EE in $\mathrm{Kcal} / \mathrm{hr}=(3.815$

$297+1.232$ X RER) X VO2 in $\mathrm{ml} / \mathrm{min}$. Indirect calorimetry data were analyzed by CALR web-based

298 software ${ }^{46}$. Body composition (fat mass) was determined using EchoMRI Body Composition

299 Analyzer. Animal experiments were conducted in accordance with the Mount Sinai and UCLA

300 Institutional Animal Care and Research Advisory Committee.

301

302 RNA-Seq

303 RNA isolation, library preparation, and analysis were conducted as previously described ${ }^{24}$. Flash304 frozen mgWAT samples were homogenized in QIAzol (Qiagen, Germantown, MD), and following 305 chloroform phase separation, RNA was isolated according to the manufacturer's protocol using 306 miRNeasy columns (Qiagen, Germantown, MD). Libraries were prepared from extracted mgWAT 307 fat RNA (Agilent 2200 Tapestation eRIN >8.2) using KAPA Stranded mRNA-Seq Kit (cat 308 \#KK8421, KAPA Biosystems, Wilmington, MA), per the manufacturers' instructions. The pooled 309 libraries were sequenced using an Illumina HiSeq4000 instrument with SE50bp reads (Illumina, 310 San Diego, CA). Reads were aligned to the mouse genome mm10 using STAR ${ }^{47}$ or HISAT2 ${ }^{48}$ 311 aligner and quantified using the Bioconductor $\mathrm{R}$ packages as described in the RNA-Seq 
312 workflow ${ }^{49} . P$ values were adjusted using the Benjamini-Hochberg procedure of multiple 313 hypothesis testing ${ }^{49}$.

\section{Single cell isolation from SVF}

316 Single cell SVF populations from adipose tissue were isolated as described previously ${ }^{3,31}$. The

317 fourth inguinal white adipose tissue (iWAT) depot mgWAT from mice exposed to cold stress $318\left(4^{\circ} \mathrm{C}\right)$ or room temperature for $24 \mathrm{hr}$ was dissected and placed on a sterile 6-well tissue culture 319 plate with ice-cold 1X DPBS. Excess liquid was removed from fat pads by blotting. Each tissue 320 was cut and minced with scissors and then placed in $15 \mathrm{ml}$ conical tubes containing digestion 321 buffer (2 $\mathrm{ml}$ DPBS and Collagenase II at $3 \mathrm{mg} / \mathrm{ml}$; Worthington Biochemical, Lakewood, NJ, 322 USA) for $40 \mathrm{~min}$ of incubation at $37^{\circ} \mathrm{C}$ with gentle shaking at $100 \mathrm{rpm}$. Following tissue digestion, 323 enzyme activity was stopped with $8 \mathrm{ml}$ of resuspension media (DMEM/F12 with glutamax 324 supplemented with $15 \%$ FBS and $1 \%$ pen/strep; Thermo Scientific, CA). The digestion mixture 325 was passed through $100 \mu \mathrm{m}$ cell strainer and centrifuged at $150 \mathrm{x} \mathrm{g}$ for $8 \mathrm{~min}$ at room temperature. 326 To remove red blood cells, the pellet was resuspended and incubated in RBC lysis buffer (Thermo 327 Scientific, CA) for $3 \mathrm{~min}$ at room temperature, followed by centrifugation at $150 \mathrm{xg}$ for $8 \mathrm{~min}$. 328 The pellet was then resuspended in resuspension media and again spun down at $150 \mathrm{xg}$ for $8 \mathrm{~min}$. 329 The cell pellet was resuspended in $1 \mathrm{ml}$ of $0.01 \%$ BSA (in DPBS) and passed through a $40 \mu \mathrm{m}$ cell 330 strainer (Fisher Scientific, Hampton, NH, USA) to discard debris. Cell number was counted for 331 10X Genomics single cell application. 
334 To yield an expected recovery of 4000-7000 single cells, an estimated 10,000 single cells per 335 channel were loaded onto Single Cell 3' Chip (10X Genomics, CA). The Single Cell 3' Chip was

336 placed on a 10X Genomics instrument to generate single cell gel beads in emulsion (GEMs).

337 Chromium Single Cell 3'v3 Library and Cell Bead Kits were used according to the manufacturer's 338 instructions to prepare single cell RNA-Seq libraries.

\section{Illumina high-throughput sequencing libraries}

341 Qubit Fluorometric Quantitation (ThermoFisher, Canoga Park, CA, USA) was used to quantify 342 the 10X Genomics library molar concentration and a TapeStation (Aligent, Santa Clara, CA, USA) 343 was used to estimated library fragment length. Libraires were pooled and sequenced on an Illumina 344 HiSeq 4000 (Illumina, San Diego, CA, USA) with PE100 reads and an 8 bp index read for 345 multiplexing. Read 1 contained the cell barcode and UMI and read 2 contained the single cell 346 transcripts.

348 Single cell data pre-processing and quality control

349 To obtain digital gene expression matrices (DGEs) in sparse matrix representation, paired end 350 reads from the Illumina HiSeq 4000 were processed and mapped to the mm10 mouse genome 351 using 10X Genomics' Cell Ranger v3.0.2 software suite. Briefly, .bcl files from the UCLA Broad 352 Stem Cell Research Center sequencing core were demultiplexed and converted to fastq format 353 using the 'mkfastq' function from Cell Ranger. Next, the Cell Ranger 'counts' function mapped 354 reads from fastq files to the mm10 reference genome and tagged mapped reads as either exonic, 355 intronic, or intergenic. Only reads which aligned to exonic regions were used in the resulting 356 DGEs. After combining all four sample DGEs into a single study DGE, we filtered out cells with 
357 (1) UMI counts $<700$ or $>30,000$, (2) gene counts $<200$ or $>8,000$, and (3) mitochondrial gene

358 ratio $>10 \%$. This filtering resulted in a dataset consisting of 42,052 genes across 12,222 cells, with

359 approximately $2,300-4,650$ cells from each sample. A median of 2,411 genes and 7,252

360 transcripts were detected per cell.

\section{Identification of cell clusters}

363 To achieve high resolution cell type identification and increased confidence in our cell type

364 clustering we brought in external publicly available single cell data from SVF and mammary

365 tissues. Specifically, we included single cell data from 9 datasets comprising 91,577 single cells

366 from the mammary gland and multiple adipose depots, across 4 different single cell platforms

367 (Table 1). These external datasets and the SVF data from this study were all independently

368 normalized using sctransform ${ }^{50}$ and integrated using Seurat ${ }^{51,52}$ v3.1.5. The single cell expression

369 profiles were projected into two dimensions using $\mathrm{UMAP}^{53}$ or $\mathrm{tSNE}^{54}$ and the Louvain ${ }^{55}$ method

370 for community detection was used to assign clusters. This integrated data was only used to identify

371 and define the cell types. All plots which are not explicitly designated as integrated with at least

372 one external dataset and all downstream analyses (e.g. differential expression analyses) were

373 conducted on non-integrated data to retain the biological effect of the cold treatment. Visualization

374 of the non-integrated data was conducted on a subsampled dataset where all samples had the same

375 number of cells to give an equal weight to each sample, however, all downstream analyses (e.g.

376 differential expression analyses) leveraged the full dataset.

\begin{tabular}{|l|l|l|l|l|l|l|}
\hline Cell \# & Tissue & Sex & Condition & Technology & Name & Source \\
\hline 22,800 & $\begin{array}{l}\text { BAT, EPI, } \\
\text { ING) }\end{array}$ & M & SVF & Drop-Seq & Broad SVF & $\begin{array}{l}\text { Broad } \\
\text { Single Cell } \\
\text { Portal }^{56}\end{array}$ \\
\hline
\end{tabular}




\begin{tabular}{|c|c|c|c|c|c|c|}
\hline 25,010 & Mammary & $\mathrm{F}$ & $\begin{array}{l}\text { NP, G, L, } \\
\text { PI }\end{array}$ & 10X Genomics & MammaryEpi & $\frac{\text { GSE106273 }}{57}$ \\
\hline 14,927 & Mammary & $\mathrm{F}$ & $\begin{array}{l}\text { NP, G, L, } \\
\text { PI }\end{array}$ & Microwell-seq & MouseCellAtlas & figshare 58,59 \\
\hline 4,481 & Mammary & $\mathrm{F}$ & $\mathrm{N} / \mathrm{A}$ & 10X Genomics & $\begin{array}{l}\text { TM.Mammary.10 } \\
\mathrm{X}\end{array}$ & figshare $^{17,60}$ \\
\hline 2,405 & Mammary & $\mathrm{F}$ & $\mathrm{N} / \mathrm{A}$ & $\begin{array}{l}\text { FACS + Smart- } \\
\text { seq2 }\end{array}$ & $\begin{array}{l}\text { TM.Mammary.FA } \\
\text { CS }\end{array}$ & figshare $^{17,60}$ \\
\hline 4,967 & $\begin{array}{l}\text { SVF } \\
\text { (BAT, } \\
\text { GAT, } \\
\text { MAT, } \\
\text { SCAT) }\end{array}$ & $\begin{array}{l}\mathrm{F} \\
\mathrm{M}\end{array}$ & $\mathrm{N} / \mathrm{A}$ & $\begin{array}{l}\text { FACS + Smart- } \\
\text { seq2 }\end{array}$ & TM.SVF.FACS & figshare $^{17,60}$ \\
\hline 3,132 & Mammary & $\mathrm{F}$ & $\begin{array}{l}\text { Age } \quad(3 \mathrm{~m}, \\
18 \mathrm{~m}, 21 \mathrm{~m})\end{array}$ & $\begin{array}{l}\text { FACS + Smart- } \\
\text { seq2 }\end{array}$ & $\begin{array}{l}\text { TS.Mammary.FA } \\
\text { CS }\end{array}$ & figshare ${ }^{18,61}$ \\
\hline 5,080 & $\begin{array}{l}\text { SVF } \\
\text { (BAT, } \\
\text { GAT, } \\
\text { MAT, } \\
\text { SCAT) }\end{array}$ & $\begin{array}{l}\mathrm{F} \\
\mathrm{M}\end{array}$ & $\begin{array}{l}\text { Age }(18 \mathrm{~m}, \\
21 \mathrm{~m}, 30 \mathrm{~m})\end{array}$ & 10X Genomics & TS.SVF.10X & figshare $^{18,61}$ \\
\hline 8,775 & $\begin{array}{l}\text { SVF } \\
\text { (BAT, } \\
\text { GAT, } \\
\text { MAT, } \\
\text { SCAT) }\end{array}$ & $\begin{array}{l}\mathrm{F} \\
\mathrm{M}\end{array}$ & $\begin{array}{l}\text { Age } \quad(3 \mathrm{~m}, \\
18 \mathrm{~m}, 24 \mathrm{~m})\end{array}$ & $\begin{array}{l}\text { FACS + Smart- } \\
\text { seq2 }\end{array}$ & TS.SVF.FACS & figshare $^{18,61}$ \\
\hline
\end{tabular}

Table 1: Publicly available single cell datasets used in this study

379 BAT - brown adipose tissue, EPI - epididymal white adipose tissue, ING - inguinal white adipose

380 tissue, NP - nulliparous, G - gestation, L - lactation, PI - post involution, GAT - gonadal adipose

381 tissue, MAT - mesenteric adipose tissue, SCAT - subcutaneous adipose tissue

383 Cell type-specific gene expression signatures

384 Cell type-specific gene expression signatures were generated by identifying genes with expression

385 levels two-fold greater (adjusted p-values $<0.05$ ) than all other cell types. To ensure consistency 386 across samples, Seurat's FindConservedMarkers function (Wilcoxon rank sum test with a meta p387 value) was applied across each sample. 
Resolving cell identities of the cell clusters

390 To identify the cell type identity of each cluster, we used a curated set of canonical marker genes 391 derived from the literature (Supplementary Table 1) to find distinct expression patterns in the

392 cell clusters. Clusters which uniquely expressed known marker genes were used as evidence to 393 identify that cell type. Cell subtypes which did not express previously established markers were 394 identified by general cell type markers and novel markers obtained with Seurat's 395 FindConservedMarkers function were used to define the cell subtype.

\section{Differential gene expression analysis}

398 Within each identified cell type, cold treated and room temperature single cells were compared for 399 differential gene expression using Seurat's FindMarkers function (Wilcoxon rank sum test) in a 400 manner similar to Li et al. ${ }^{19}$. Differentially expressed genes were identified using two criteria: (i) 401 an expression difference of $>=1.5$-fold and adjusted $p$-value $<0.05$ in a grouped analysis between 402 room temperature mice $(n=2)$ and cold treated mice $(n=2)$; (ii) an expression difference of $>=$ 4031.25 fold and consistent fold change direction in all 4 possible pairwise combinations of cold404 treated vs room temperature mice.

405

406 Pathway enrichment analysis

407 Pathway enrichment analysis was conducted on the differentially expressed genes from each cell 408 type using gene sets from KEGG ${ }^{62}$, Reactome ${ }^{63}$, BIOCARTA ${ }^{64}$, GO Molecular Functions ${ }^{65}$, and 409 GO Biological Processes ${ }^{65}$. Prior to enrichment, mouse gene names were converted to human 410 orthologues. Enrichment of pathways was assessed with a Fisher's exact test, followed by multiple 
411 testing correction with the Benjamini-Hochberg method. Gene set enrichments with FDR $<0.05$

412 were considered statistically significant.

413

\section{Real time qPCR}

415 Total RNA was isolated using TRIzol reagent (Invitrogen) and reverse transcribed with the iScript 416 cDNA synthesis kit (Biorad). cDNA was quantified by real-time PCR using SYBR Green Master 417 Mix (Diagenode) on a QuantStudio 6 instrument (Themo Scientific, CA). Gene expression levels 418 were determined by using a standard curve. Each gene was normalized to the housekeeping gene 419 36B4 and was analyzed in duplicate. Primers used for real-time PCR are previously described 3,31 420 and presented in Table 2.

421

422 RNAScope Fluorescence in situ hybridization (FISH)

423 mgWAT from RT or cold exposed mice (Jackson Laboratory, \#000664) was fixed in 10\% formalin 424 overnight, embedded with paraffin, and sectioned into unstained, $5 \mu \mathrm{m}$-thick sections. Sections 425 were baked at $60^{\circ} \mathrm{C}$ for 1 hour, deparaffinized, and baked again at $60^{\circ} \mathrm{C}$ for another hour prior to 426 pre-treatment. The standard pre-treatment protocol was followed for all sectioned tissues. In situ 427 hybridization was performed according to manufacturer's instructions using the RNAscope 428 Multiplex Fluorescent Reagent Kit v2 (\#323136, Advanced Cell Diagnostics [ACD], Newark, 429 CA). Opal fluorophore reagent packs (Akoya Biosciences, Menlo Park, CA) for Opal 520 430 (FP1487A), Opal 570 (FP1488A), Opal 620 (FP1495A), and Opal 690 (FP1497A) were used at a 431 1:1000 dilution in TSA buffer (\#322809, ACD). RNAscope probes from ACD were used for the 432 following targets: EPCAM (\#418151), ENHO (\#873251), LRG1 (\#423381), LCN2 (\#313971), HP 433 (\#532711), WNT4 (\#401101), NRG4 (\#493731), KRT8 (\#424528), and MFGE8 (\#408778). 
434 Slides were mounted with ProLong Diamond Antifade Mountant with DAPI (P36962, Life

435 Technologies, Carlsbad, CA). Fluorescent signals were captured with the 40x objective lens on a 436 laser scanning confocal microscope LSM880, (Zeiss, White Plains, NY).

438 Fluorescent-activated cell sorting (FACS)

439 Mammary gland white adipose tissue (mgWAT) from RT or cold exposed mice (Jackson 440 Laboratory, \#000664) was dissected, cut, minced, and digested with collagenase D (5 mg/mL, 441 \#11088882001, Roche, Germany) and dispase (2 mg/mL, \#17105041, Gibco, Grand Island, NY)

442 over $40 \mathrm{~min}$ at $37^{\circ} \mathrm{C}$ with gentle shaking at $100 \mathrm{RPM}$. Enzymatic digestion was stopped with $443 \mathrm{DMEM} / 15 \% \mathrm{FBS}$ and the cell suspension was filtered through a $100 \mu \mathrm{m}$ nylon mesh cell strainer, 444 and centrifuged for 10 minutes at $700 \mathrm{x}$ g. SVF pellet was resuspended in $1 \mathrm{~mL}$ Red Blood Cell 445 lysis buffer (\#41027700, Roche, Germany) and incubated for 5 minutes at room temperature. Cell 446 suspension was diluted in $4 \mathrm{~mL}$ DPBS and filtered through a $40 \mu \mathrm{m}$ nylon mesh cell strainer and 447 centrifuged for 10 minutes at $700 \mathrm{x}$ g. Single cell suspension was blocked for 10 minutes on ice in $448500 \mu \mathrm{L}$ DPBS/5\% BSA (blocking buffer), centrifuged for $10 \mathrm{~min}$ at $700 \mathrm{x}$, resuspended in 200 $449 \mu \mathrm{L}$ of DBPS/0.5\% BSA (FACS buffer) solution containing the desired antibody mix, and 450 incubated for 1 hour at $4^{\circ} \mathrm{C}$ in the dark with gentle rotation. Antibody-stained samples were washed 451 with $800 \mu \mathrm{L}$ FACS buffer, centrifuged 10 minutes at $700 \mathrm{x}$ g, and resuspended in FACS buffer 452 containing DAPI (at $1 \mathrm{ug} / \mathrm{mL}$ ). Flow cytometry analysis was performed on a BD FACS Canto II 453 (BD Biosciences, San Jose, CA) and results analyzed on FCS Express software (DeNovo Software, 454 Pasadena, CA). Fluorescently-tagged anti-mouse antibodies (BioLegend, San Diego, CA) were 455 used to label cell surface markers for flow cytometry analysis: EPCAM-FITC (clone G8.8, 456 \#118207), Sca-1-APC (Ly6, clone E13-161.7, \#122512), CD49f-APC (clone GoH3, \#313616). 
457 For flow cytometry analysis, negative selection of CD45-expressing cells using CD45 microbeads 458 (\#130052301) was performed immediately prior to the EPCAM positive selection protocol 459 described above.

461 Isolation, selection, and ex vivo treatment of EPCAM-positive cells

462 MACS microbeads (Miltenyi Biotec, Auburn, CA) were used for immuno-magnetic labeling 463 positive selection of EPCAM-expressing cells (anti-CD326, \#130105958). Before magnetic 464 labeling, a single-cell suspension from the stromal vascular fraction of female mouse iWAT was 465 prepared in MACS buffer, i.e. PBS, pH 7.2, 0.5\% bovine serum albumin (\#A7030, SIGMA, St. 466 Louis, MO) and $2 \mathrm{mM}$ EDTA, filtered through a MACS pre-separation $30 \mu \mathrm{m}$ nylon mesh 467 (\#130041407) to remove cell clumps. Then, for magnetic labeling of EPCAM-expressing cells, 10 $468 \mu \mathrm{L}$ of EPCAM microbeads were added per $1 \times 10^{7}$ total cells in $100 \mathrm{uL}$ buffer, incubated for 15 469 minutes with rotation at $4^{\circ} \mathrm{C}$, washed with $1 \mathrm{~mL}$ buffer, centrifuged at $700 \times \mathrm{g}$ for 5 minutes, 470 resuspended in $500 \mu \mathrm{L}$ buffer, and added to a pre-equilibrated MACS LS column (\#130042401) 471 in the magnetic field of a MACS separator (\#130042302). Unlabeled EPCAM-negative cells were 472 collected in the flow-through with three subsequent washes. The column was removed from the 473 magnetic field, $5 \mathrm{~mL}$ of MACS buffer were added to the column, and the magnetically-labeled 474 EPCAM-positive cells retained in the column were collected by flushing the cells down the column 475 with a plunger. Finally, EPCAM-negative and EPCAM-positive cell populations were centrifuged 476 at $700 \mathrm{x} g$ for 5 minutes, resuspended in DMEM/F12 with glutamax supplemented with $15 \%$ FBS 477 and 1\% pen/strep (Thermo Scientific, CA) and plated on Collagen I-coated 12-well tissue culture 478 plates (\#354500, Corning, Kennebunk, ME). Media was replaced every other day during 6 days, 479 followed by cell lysis with Tryzol for phenol/chloroform RNA extraction, and RT-qPCR analysis. 


\section{Adipocyte differentiation and treatments}

$48110 \mathrm{~T} 1 / 2$ or SVF from the $4^{\text {th }}$ inguinal (iWAT) mgWAT was isolated from 8 week old female Lcn2-

482 null mice, respectively. 10T1/2 cells were maintained as previously described ${ }^{3}$. The pre-iWAT

483 cells were maintained in Dulbecco's Modified Eagle Medium: Nutrient Mixture F-12

484 (DMEM/F12) supplemented with 1\% glutamax, 10\% fetal calf serum and $100 \mathrm{U} / \mathrm{ml}$ of both

485 penicillin and streptomycin (basal media). Two days after plating (day 0), when the cells reached

486 nearly $100 \%$ confluency, the cells were treated with an induction media containing basal media

487 supplemented with $4 \mu \mathrm{g} / \mathrm{mL}$ insulin, $0.5 \mathrm{mM}$ IBMX, $1 \mu \mathrm{M}$ dexamethasone, and $1 \mu \mathrm{M}$

488 rosiglitazone. After $48 \mathrm{~h}$, the cells were treated with a maintenance media containing the basal

489 media supplemented with $4 \mu \mathrm{g} / \mathrm{mL}$ insulin, and $1 \mu \mathrm{M}$ rosiglitazone, with a media change every 2

490 days until day 10. For qPCR, differentiated iWAT cells were treated with $1 \mu \mathrm{g} / \mathrm{ml}$ recombinant

491 LCN2 (Sino Biological Inc.) or differentiated 10T1/2 cells were treated with LRG1 (R\&D

492 Systems) for $24 \mathrm{~h}$ and then treated with isoproterenol (Sigma) for $6 \mathrm{~h}$ after which RNA was 493 collected.

494

495 iDISCO and Adipoclear tissue labelling and clearing

496 Sample collection

497 Immediately after cold exposure mice were anaesthetized with isoflurane (3\%) and perfused with 498 heparinized saline followed by 4\% paraformaldefyde (PFA) (Electron Microscopy Sciences, 499 Hatfield, PA, USA). Fat pads were carefully dissected and postfixed overnight in $4 \%$ PFA at $4^{\circ} \mathrm{C}$. 500 On the following day the tissue was washed 3 times in PBS before proceeding with the optical 501 clearing protocol. 


\section{Optical clearing}

504 Whole-mount staining and clearing was performed using the Adipo-Clear protocol as previously

505 described $^{1}$. Dissected fat pads were dehydrated at room temperature (RT) with a methanol/B1n

506 buffer (0.3 M glycine, $0.1 \%$ Triton $\mathrm{X}-100$ in $\left.\mathrm{H}_{2} \mathrm{O}, \mathrm{pH} 7\right)$ gradient $(20 \%, 40 \%, 60 \%, 80 \%, 100 \%)$,

507 incubated 3 times (1h, o/n, 2h) in 100\% dichloromethane (DCM) (Sigma-Aldrich, St. Louis, MO,

508 USA) to remove hydrophobic lipids, washed twice in 100\% methanol, and bleached in $5 \%$

$509 \mathrm{H}_{2} \mathrm{O}_{2}$ overnight at $4^{\circ} \mathrm{C}$ to reduce tissue autofluorescence. Fat pads were then rehydrated with a 510 methanol/B1n buffer gradient $(80 \%, 60 \%, 40 \%, 20 \%)$ and then washed twice in 100\% B1n buffer 511 (1h, o/n). Primary antibodies (EPCAM: 1:250; TH: 1:500) were diluted in modified PTxwH (PBS

512 with $0.5 \%$ Trixon X-100, $0.1 \%$ Tween-20, $2 \mu \mathrm{g} / \mathrm{ml}$ heparin, as previously described ${ }^{66}$ and applied

513 for 6 days at $37^{\circ} \mathrm{C}$. Following 5 washes with modified PTxwH over 1 day with the last wash

514 performed overnight, secondary antibodies were diluted in modified PTxwH (1:500) and samples

515 incubated for 6 days at $37^{\circ} \mathrm{C}$. Samples were washed 5 times over 1 day in modified PTxwH at $37^{\circ} \mathrm{C}$,

5165 times over 1 day in PBS at RT, embedded in 1\% agarose, dehydrated with a methanol gradient

517 in $\mathrm{H}_{2} \mathrm{O}(12 \%, 50 \%, 75 \%, 100 \%)$, washed 3 times for $1 \mathrm{hr}$ in $100 \%$ methanol followed by 3 times

518 for $1 \mathrm{hr}$ in DCM, before being transferred to dibenzylether (DBE) (Sigma-Aldrich) to clear.

$519 \quad$ Imaging

520 Z-stacked optical sections of whole fat pads were acquired with an Ultramicroscope II (LaVision

521 BioTec, Bielefeld, Germany) at a 1.3x magnification with a $4 \mu \mathrm{m}$ step size and dynamic focus

522 with a maximum projection filter. Samples were then imaged in glass-bottom $\mu$-dishes $(81158$,

523 Ibidi, Gräfelfing, Germany) using an inverted Zeiss LSM 710 confocal microscope with a 10x

524 (NA: 0.3) objective and a step size of $5 \mu \mathrm{m}$.

525 


\section{Image analysis}

527 Imaris versions 9.6.0-9.7.2 (Bitplane AG, Zürich, Switzerland) were used to create digital surfaces

528 covering ducts, TH+ innervation and total sample volume (1.3x light sheet images and 10x

529 confocal images) to automatically determine volumes and intensity data. Volume reconstructions

530 were performed using the surface function with local contrast background subtraction. For 531 detection of EPCAM+ ducts in 1.3x light sheet images, a smoothing factor of $5 \mu \mathrm{m}$ was used and 532 the threshold factor was set to correspond to the largest duct diameter in each sample. For detection 533 of $\mathrm{TH}+$ nerves in $1.3 \mathrm{x}$ light sheet images, a smoothing factor of $3 \mu \mathrm{m}$ and a threshold factor of 80 $534 \mu \mathrm{m}$ were used. For detection of EPCAM+ ducts in 10x confocal images, a smoothing factor of $5353.35 \mu \mathrm{m}$ was used and a threshold factor corresponding to the diameter of the thickest duct wall in 536 each sample was used. For detection of TH+ nerves in 10x confocal images, a smoothing factor of $5372 \mu \mathrm{m}$ and a threshold factor of $5 \mu \mathrm{m}$ were used. In 10x confocal images, nerve/duct interactions 538 were defined by masking the $\mathrm{TH}$ channel using the $\mathrm{TH}+$ nerve surface to remove any background, 539 and then masking it again using the EPCAM+ duct surface. This process revealed TH+ innervation 540 overlapping with EPCAM+ staining. A new surface was created to cover this overlapping TH+ 541 innervation using a smoothing factor of $2 \mu \mathrm{m}$ and a threshold factor of $5 \mu \mathrm{m}$.

\section{$542 \quad$ Statistical analyses}

543 Data are shown as mean \pm S.E.M. Distribution was assessed by Shapiro-Wilk test. Significance was 544 determined by a two-tailed unpaired $t$ test (parametric distribution) or by a Mann-Whitney test 545 (non-parametric distribution). Significance was set at an alpha level of 0.05.

\section{Mammary gland organoids culture}

547 For organoid culture, we used a previously published protocol ${ }^{67}$. In brief, fat pads of 8-9-week548 old K8rtTA/TetO-DTA ${ }^{39}$ virgin female mice were dissected and the lymph nodes removed. 
549 Tissues were briefly washed in $70 \%$ ethanol and manually chopped into $1 \mathrm{~mm}^{3}$ pieces. The finely 550 minced tissue was transferred to a digestion mix consisting of serum-free Leibovitz's L15 medium

551 (Gibco) containing $3 \mathrm{mg} \mathrm{ml}^{-1}$ collagenase A (Sigma) and $1.5 \mathrm{mg} \mathrm{ml}^{-1}$ trypsin (Sigma). This was 552 incubated for $1 \mathrm{hr}$ at $37^{\circ} \mathrm{C}$ to liberate epithelial tissue fragments ('organoids'). Isolated organoids 553 were mixed with $50 \mu \mathrm{l}$ of phenol-red-free Matrigel (BD Biosciences) and seeded in 24-well plates.

554 The basal culture medium contained phenol-red-free DMEM/F-12 with penicillin/streptomycin, $55510 \mathrm{mM}$ HEPES (Invitrogen), Glutamax (Invitrogen), N2 (Invitrogen) and B27 (Invitrogen). The 556 basal medium was supplemented with Nrg1 (100 ng ml $\left.{ }^{-1}, \mathrm{R} \& \mathrm{D}\right)$, Noggin (100 ng ml ${ }^{-1}$, Peprotech) 557 and R-spondin 1 (100 $\mathrm{ng} \mathrm{m}^{-1}$, R\&D). Then, $500 \mu$ l supplemented basal culture medium was added 558 per well and organoids were maintained in a $37^{\circ} \mathrm{C}$ humidified atmosphere under $5 \% \mathrm{CO}_{2}$. After 559 one week in culture, mammary organoids were released from the Matrigel by breaking the matrix 560 with a P1000 pipette on ice. After 2-3 passages of washing and centrifugation at 1,500 rpm (140g)

561 for $5 \mathrm{~min}$ at $4{ }^{\circ} \mathrm{C}$, mammary cells were resuspended in Matrigel, seeded in 24-well plates and 562 exposed to the previously described culture conditions. Organoids were treated either with $10 \mu \mathrm{g}$ $563 \mathrm{ml}^{-1}$ of DOX to promote luminal cell ablation or with $10 \mu \mathrm{M}$ of isoproterenol (Sigma) for $6 \mathrm{hr}$. 564 After $6 \mathrm{hr}$ treatment, organoids were collected from Matrigel as mentioned before to perform 565 further analysis.

\section{RNA extraction and quantitative real-time PCR in organoid samples}

567 To perform RNA extraction, isolated organoids were collected into kit lysis buffer. RNA was 568 extracted with Qiagen RNeasy Micro Kit. After nanodrop RNA quantification and analysis of 569 RNA integrity, purified RNA was used to synthesize the first-strand cDNA in a $30 \mu$ final volume, 570 using Superscript II (Invitrogen) and random hexamers (Roche). Genomic contamination was 571 detected by performing the same procedure without reverse transcriptase. Quantitative PCR 
572 analyses were performed with $1 \mathrm{ng}$ of cDNA as template, using FastStart Essential DNA green 573 master (Roche) and a Light Cycler 96 (Roche) for real-time PCR system. Relative quantitative 574 RNA was normalized using the housekeeping gene Gapdh. Analysis of the results was performed 575 using Light Cycler 96 software (Roche) and relative quantification was performed using the $\mathrm{ddCt}$ 576 method using Gapdh as a reference.

\section{Immunofluorescence in organoid samples}

579 For immunofluorescence, collected organoids were pre-fixed in 4\% PFA for 30 min at RT. Pre580 fixed organoids were washed in $2 \%$ FBS-PBS, embedded in OCT and kept at $-80^{\circ} \mathrm{C}$. Sections of 4 $581 \mu \mathrm{m}$ were cut using a HM560 Microm cryostat (Mikron Instruments). Tissue sections were 582 incubated in blocking buffer (BSA 1\%, HS 5\%, Triton-X 0.2\% in PBS) for $1 \mathrm{hr}$ at RT. The different 583 primary antibodies were incubated overnight at $4{ }^{\circ} \mathrm{C}$. Sections were then rinsed in PBS and 584 incubated with the corresponding secondary antibodies diluted at 1:400 in blocking buffer for $1 \mathrm{hr}$ 585 at RT. The following primary antibodies were used: rat anti-K8 (1:1,000, Troma-I, Developmental 586 Studies Hybridoma Bank, University of Iowa), rabbit anti-EPCAM (1:1,000, ab71916, Abcam), 587 goat anti-Len2 (1:50, AF1857, R\&D). The following secondary antibodies, diluted 1:400, were 588 used: anti-goat (A11055) conjugated to Alexa Fluor 488 (Invitrogen), anti-rat (712-295-155) 589 rhodamine Red-X and anti-rabbit (711-605-152) Cy5 (Jackson ImmunoResearch). Nuclei were 590 stained with Hoechst solution $(1: 2,000)$ and slides were mounted in DAKO mounting medium 591 supplemented with 2.5\% DABCO (Sigma).

593 Conflict of Interests (COI)

594 AJB is a co-founder and consultant to Personalis and NuMedii; consultant to Samsung, Mango 595 Tree Corporation, and in the recent past, $10 \times$ Genomics, Helix, Pathway Genomics, and Verinata 
596 (Illumina); has served on paid advisory panels or boards for Geisinger Health, Regenstrief 597 Institute, Gerson Lehman Group, AlphaSights, Covance, Novartis, Genentech, and Merck, and 598 Roche; is a shareholder in Personalis and NuMedii; is a minor shareholder in Apple, Facebook, 599 Alphabet (Google), Microsoft, Amazon, Snap, $10 \times$ Genomics, Illumina, CVS, Nuna Health, 600 Assay Depot, Vet24seven, Regeneron, Sanofi, Royalty Pharma, AstraZeneca, Moderna, Biogen,

601 Paraxel, and Sutro, and several other non-health related companies and mutual funds; and has 602 received honoraria and travel reimbursement for invited talks from Johnson and Johnson, Roche, 603 Genentech, Pfizer, Merck, Lilly, Takeda, Varian, Mars, Siemens, Optum, Abbott, Celgene, 604 AstraZeneca, AbbVie, Westat, and many academic institutions, medical or disease specific 605 foundations and associations, and health systems. AJB receives royalty payments through Stanford 606 University, for several patents and other disclosures licensed to NuMedii and Personalis. AJB's 607 research has been funded by NIH, Northrup Grumman (as the prime on an NIH contract), 608 Genentech, Johnson and Johnson, FDA, Robert Wood Johnson Foundation, Leon Lowenstein 609 Foundation, Intervalien Foundation, Priscilla Chan and Mark Zuckerberg, the Barbara and Gerson 610 Bakar Foundation, and in the recent past, the March of Dimes, Juvenile Diabetes Research 611 Foundation, California Governor's Office of Planning and Research, California Institute for 612 Regenerative Medicine, L'Oreal, and Progenity.

613 SAS is a named inventor of the intellectual property, "Compositions and Methods to Modulate 614 Cell Activity", a co-founder of and has equity in the private company Redpin Therapeutics.

615 The rest of the authors declare no COIs.

\section{Acknowledgements}

617 A.A is supported by senior postdoctoral fellowship from the Charles H. Revson Foundation (grant 618 no. 18-25), a fellowship from Sweden-America Foundation (Ernst O Eks fond), and a postdoctoral 
619 scholarship from the Swedish Society for Medical Research (SSMF). S.A.S is supported by

620 American Diabetes Association Pathway to Stop Diabetes Grant ADA \#1-17-ACE-31 NIH

621 (R01NS097184, OT2OD024912, and R01DK124461) Department of Defense (W81XWH-20-1-

622 0345, W81XWH-20-1-0156). A.J.L is supported by NIH U01 AG070959 and U54 DK120342.

623 P.R is supported by R00DK114571, NIDDK-supported Einstein-Sinai Diabetes Research Center

624 (DRC) Pilot \& Feasibility Award, and Diabetes Research and Education Foundation (DREF) Grant

625 \# 501 (PR). The funders had no role in study design, data collection and interpretation, or the

626 decision to submit the work for publication.

627

628 Contributions

629 D.A. performed all the scRNA-seq data analysis under the supervision of X.Y. L.C.S. performed 630 most of the biological experiments under the supervision of P.R. A.A. performed iDISCO and data 631 analysis under the supervision of S.A.S. K.C.K performed LCN2 related animal experiments under 632 the supervision of A.J.L. A.C.S performed organoid experiments under the supervision of C.B.

633 S.P. performed RNAscope and EPCAM cells isolation experiments under supervision of L.C.S 634 and P.R. S.S. performed indirect calorimetry and body composition studies under the supervision 635 of P.R.. I.S.A, G.D., and I.C., prepared single cell suspensions of mgWAT SVFs under the 636 supervision of P.R. and X.Y.. P.R. conceived the project and wrote the manuscript with help from 637 A.J.B., C.B., S.A.S, A.J.L., and X.Y. 


\section{REFERENCES}

6411 Chi, J. et al. Three-Dimensional Adipose Tissue Imaging Reveals Regional Variation in

642 Beige Fat Biogenesis and PRDM16-Dependent Sympathetic Neurite Density. Cell Metab 643 27, 226-236 e223, doi:10.1016/j.cmet.2017.12.011 (2018).

6442 Zhang, F. et al. An Adipose Tissue Atlas: An Image-Guided Identification of Human-like 645 BAT and Beige Depots in Rodents. Cell Metab 27, 252-262.e253, 646 doi:10.1016/j.cmet.2017.12.004 (2018).

6473 Rajbhandari, P. et al. IL-10 Signaling Remodels Adipose Chromatin Architecture to Limit 648 Thermogenesis and Energy Expenditure. Cell 172, 218-233 e217, doi:10.1016/j.cell.2017.11.019(2018).

651

652

4 Knights, A. J., Wu, J. \& Tseng, Y.-H. The Heating Microenvironment: Intercellular Cross Talk Within Thermogenic Adipose Tissue. Diabetes 69, 1599, doi:10.2337/db20-0303 (2020).

6535 Li, J. et al. Neurotensin is an anti-thermogenic peptide produced by lymphatic endothelial cells. Cell Metab 33, 1449-1465.e1446, doi:10.1016/j.cmet.2021.04.019 (2021). $\mathrm{Hu}$, B. et al. $\gamma \delta \mathrm{T}$ cells and adipocyte IL-17RC control fat innervation and thermogenesis. Nature 578, 610-614, doi:10.1038/s41586-020-2028-z (2020). Wang, Q. et al. IL-27 signalling promotes adipocyte thermogenesis and energy expenditure. Nature, doi:10.1038/s41586-021-04127-5 (2021).

8 Gjorevski, N. \& Nelson, C. M. Integrated morphodynamic signalling of the mammary gland. Nat Rev Mol Cell Biol 12, 581-593, doi:10.1038/nrm3168 (2011).

9 McNally, S. \& Martin, F. Molecular regulators of pubertal mammary gland development. Ann Med 43, 212-234, doi:10.3109/07853890.2011.554425 (2011).

10 Inman, J. L., Robertson, C., Mott, J. D. \& Bissell, M. J. Mammary gland development: cell fate specification, stem cells and the microenvironment. Development 142, 1028-1042, doi:10.1242/dev.087643 (2015).

11 Wang, Q. A. \& Scherer, P. E. Remodeling of Murine Mammary Adipose Tissue during Pregnancy, Lactation, and Involution. J Mammary Gland Biol Neoplasia 24, 207-212, doi:10.1007/s10911-019-09434-2 (2019).

12 Landskroner-Eiger, S., Park, J., Israel, D., Pollard, J. W. \& Scherer, P. E. Morphogenesis of the developing mammary gland: stage-dependent impact of adipocytes. Dev Biol 344, 968-978, doi:10.1016/j.ydbio.2010.06.019 (2010).

13 Wang, Q. A. et al. Reversible De-differentiation of Mature White Adipocytes into Preadipocyte-like Precursors during Lactation. Cell Metab 28, 282-288 e283, doi:10.1016/j.cmet.2018.05.022 (2018). transcriptomic data across different conditions, technologies, and species. Nat Biotechnol 36, 411-420, doi:10.1038/nbt.4096 (2018).

67815 Arneson, D. et al. Single cell molecular alterations reveal target cells and pathways of concussive brain injury. Nat Commun 9, 3894, doi:10.1038/s41467-018-06222-0 (2018). Bach, K. et al. Differentiation dynamics of mammary epithelial cells revealed by singlecell RNA sequencing. Nat Commun 8, 2128, doi:10.1038/s41467-017-02001-5 (2017). 
68418 Tabula Muris, C. A single-cell transcriptomic atlas characterizes ageing tissues in the mouse. Nature 583, 590-595, doi:10.1038/s41586-020-2496-1 (2020).

19 Li CM, S. H., Tsiobikas C, Selfors L, Chen H, Gray GK, Oren Y, Pinello L, Regev A, Brugge JS. Aging-associated alterations in the mammary gland revealed by single-cell RNA sequencing. bioRxiv (2019).

20 Li, C. M.-C. et al. Aging-Associated Alterations in Mammary Epithelia and Stroma Revealed by Single-Cell RNA Sequencing. Cell Reports 33, 108566, doi:https://doi.org/10.1016/j.celrep.2020.108566 (2020). Aryal, B. et al. Absence of ANGPTL4 in adipose tissue improves glucose tolerance and attenuates atherogenesis. JCI Insight 3, doi:10.1172/jci.insight.97918 (2018).

22 De Keyser, J., De Backer, J. P., Ebinger, G. \& Vauquelin, G. Regional distribution of the dopamine D2 receptors in the mesotelencephalic dopamine neuron system of human brain. J Neurol Sci 71, 119-127, doi:10.1016/0022-510x(85)90041-3 (1985).

23 Arner, E. et al. Ceruloplasmin is a novel adipokine which is overexpressed in adipose tissue of obese subjects and in obesity-associated cancer cells. PLoS One 9, e80274, doi:10.1371/journal.pone.0080274 (2014).

24 Chella Krishnan, K. et al. Sex-specific metabolic functions of adipose Lipocalin-2. Mol Metab 30, 30-47, doi:10.1016/j.molmet.2019.09.009 (2019).

25 Sun, W. Y. et al. Lipocalin-2 derived from adipose tissue mediates aldosterone-induced renal injury. JCI Insight 3, doi:10.1172/jci.insight.120196 (2018).

26 Deis, J. A. et al. Adipose Lipocalin 2 overexpression protects against age-related decline in thermogenic function of adipose tissue and metabolic deterioration. Mol Metab 24, 1829, doi:10.1016/j.molmet.2019.03.007 (2019).

27 Ishii, A. et al. Obesity-promoting and anti-thermogenic effects of neutrophil gelatinaseassociated lipocalin in mice. Sci Rep 7, 15501, doi:10.1038/s41598-017-15825-4 (2017).

28 Wang, G. X. et al. The brown fat-enriched secreted factor Nrg4 preserves metabolic homeostasis through attenuation of hepatic lipogenesis. Nat Med 20, 1436-1443, doi:10.1038/nm.3713 (2014).

29 Maffei, M., Barone, I., Scabia, G. \& Santini, F. The Multifaceted Haptoglobin in the Context of Adipose Tissue and Metabolism. Endocr Rev 37, 403-416, doi:10.1210/er.2016-1009 (2016).

30 Kumar, K. G. et al. Identification of adropin as a secreted factor linking dietary macronutrient intake with energy homeostasis and lipid metabolism. Cell Metab 8, 468481, doi:10.1016/j.cmet.2008.10.011 (2008).

31 Rajbhandari, P. et al. Single cell analysis reveals immune cell-adipocyte crosstalk regulating the transcription of thermogenic adipocytes. Elife 8, doi:10.7554/eLife.49501 (2019).

32 Gargiulo, L. et al. A Novel Effect of beta-Adrenergic Receptor on Mammary Branching Morphogenesis and its Possible Implications in Breast Cancer. J Mammary Gland Biol Neoplasia 22, 43-57, doi:10.1007/s10911-017-9371-1 (2017).

33 Marchetti, B. et al. Beta-adrenergic receptors in the rat mammary gland during pregnancy and lactation: characterization, distribution, and coupling to adenylate cyclase. Endocrinology 126, 565-574, doi:10.1210/endo-126-1-565 (1990). 
35 Gargiulo, L. et al. A Novel Effect of $\beta$-Adrenergic Receptor on Mammary Branching Morphogenesis and its Possible Implications in Breast Cancer. J Mammary Gland Biol Neoplasia 22, 43-57, doi:10.1007/s10911-017-9371-1 (2017).

36 Feng, Y., Manka, D. R., Wagner, K.-U. \& Khan, S. A. Estrogen receptor0 \pm expression in the mammary epithelium is required for ductal and alveolar morphogenesis in mice. Proceedings of the National Academy of Sciences 104, 14718 - 14723 (2007).

37 Lawson, D. A., Werb, Z., Zong, Y. \& Goldstein, A. S. The Cleared Mammary Fat Pad Transplantation Assay for Mammary Epithelial Organogenesis

38 Chi, J. et al. Three-Dimensional Adipose Tissue Imaging Reveals Regional Variation in Beige Fat Biogenesis and PRDM16-Dependent Sympathetic Neurite Density. Cell Metab 27, 226-236.e223, doi:10.1016/j.cmet.2017.12.011 (2018).

39 Centonze, A. et al. Heterotypic cell-cell communication regulates glandular stem cell multipotency. Nature 584, 608-613, doi:10.1038/s41586-020-2632-y (2020).

40 Bauters, D., Van Hul, M. \& Lijnen, H. R. Macrophage elastase (MMP-12) in expanding murine adipose tissue. Biochim Biophys Acta 1830, 2954-2959, doi:10.1016/j.bbagen.2012.12.024 (2013).

41 Lee, J. T. et al. Macrophage metalloelastase (MMP12) regulates adipose tissue expansion, insulin sensitivity, and expression of inducible nitric oxide synthase. Endocrinology 155, 3409-3420, doi:10.1210/en.2014-1037 (2014).

42 Shao, M. et al. Zfp423 Maintains White Adipocyte Identity through Suppression of the Beige Cell Thermogenic Gene Program. Cell Metab 23, 1167-1184, doi:10.1016/j.cmet.2016.04.023 (2016).

43 Gavalda-Navarro, A. et al. Lipopolysaccharide-binding protein is a negative regulator of adipose tissue browning in mice and humans. Diabetologia 59, 2208-2218, doi:10.1007/s00125-016-4028-y (2016).

44 Sun, W. et al. snRNA-seq reveals a subpopulation of adipocytes that regulates thermogenesis. Nature 587, 98-102, doi:10.1038/s41586-020-2856-x (2020).

45 Kiefer, F. W. et al. Retinaldehyde dehydrogenase 1 regulates a thermogenic program in white adipose tissue. Nat Med 18, 918-925, doi:10.1038/nm.2757 (2012).

46 Mina, A. I. et al. CalR: A Web-Based Analysis Tool for Indirect Calorimetry Experiments. Cell Metab 28, 656-666 e651, doi:10.1016/j.cmet.2018.06.019 (2018).

47 Dobin, A. et al. STAR: ultrafast universal RNA-seq aligner. Bioinformatics 29, 15-21, doi:10.1093/bioinformatics/bts635 (2013).

48 Kim, D., Langmead, B. \& Salzberg, S. L. HISAT: a fast spliced aligner with low memory requirements. Nat Methods 12, 357-360, doi:10.1038/nmeth.3317 (2015).

49 Love, M. I., Anders, S., Kim, V. \& Huber, W. RNA-Seq workflow: gene-level exploratory analysis and differential expression. Fl000Res 4, 1070, doi:10.12688/f1000research.7035.1 (2015).

50 Hafemeister, C. \& Satija, R. Normalization and variance stabilization of single-cell RNAseq data using regularized negative binomial regression. Genome Biol 20, 296, doi:10.1186/s13059-019-1874-1 (2019).

773 transcriptomic data across different conditions, technologies, and species. Nature Biotechnology 36, 411-420, doi:10.1038/nbt.4096 (2018). 
77552 Stuart, T. et al. Comprehensive Integration of Single-Cell Data. Cell 177, 1888776 1902.e1821, doi:10.1016/j.cell.2019.05.031 (2019).

77753 McInnes, L., Healy, J. \& Melville, J. UMAP: Uniform Manifold Approximation and $778 \quad$ Projection for Dimension Reduction. arXiv (2018).

$77954 \quad$ van der Maaten, L. \& Hinton, G. Visualizing Data using t-SNE. J Mach Learn Res, 25792605 (2008).

55 Blondel, V. D., Guillaume, J.-L., Lambiotte, R. \& Lefebvre, E. Fast unfolding of communities in large networks. J. Stat. Mech. 2008, P10008, doi:10.1088/17425468/2008/10/P10008 (2008).

56 Broad Institute. Study: Mouse Adipose Stromal Vascular Fraction, $<$ https://singlecell.broadinstitute.org/single_cell/study/SCP708/mouse-adipose-stromalvascular-fraction\#study-summary $>$ (

57 Bach, K. et al. Differentiation dynamics of mammary epithelial cells revealed by singlecell RNA sequencing. Nature Communications 8, 2128, doi:10.1038/s41467-017-02001-5 (2017).

58 Guo, G. MCA DGE Data. Figshare, doi:10.6084/m9.figshare.5435866.v8 (2020).

79159 Han, X. et al. Mapping the Mouse Cell Atlas by Microwell-Seq. Cell 172, 10911107.e1017, doi:10.1016/j.cell.2018.02.001 (2018).

60 Tabula Muris: Transcriptomic characterization of 20 organs and tissues from Mus musculus at single cell resolution.

79561 Tabula Muris Senis.

79662 Kanehisa, M. \& Goto, S. KEGG: Kyoto Encyclopedia of Genes and Genomes. Nucleic Acids Res 28, 27-30, doi:10.1093/nar/28.1.27 (2000).

63 Jassal, B. et al. The Reactome Pathway Knowledgebase. Nucleic Acids Res 48, D498D503, doi:10.1093/nar/gkz1031 (2020).

80064 Nishimura, D. BioCarta. Biotech Software \& Internet Report 2, 117-120, doi:10.1089/152791601750294344 (2001).

65 Ashburner, M. et al. Gene Ontology: tool for the unification of biology. Nat Genet 25, 2529, doi:10.1038/75556 (2000).

66 Alvarsson, A. et al. Optical Clearing and 3D Analysis Optimized for Mouse and Human Pancreata. Bio Protoc 11, e4103, doi:10.21769/BioProtoc.4103 (2021).

67 Jardé, T. et al. Wnt and Neuregulin1/ErbB signalling extends 3D culture of hormone responsive mammary organoids. Nature Communications 7, 13207, doi:10.1038/ncomms13207 (2016). 
A

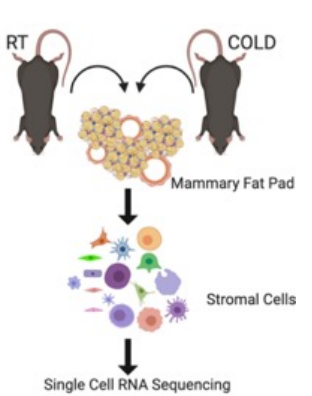

D

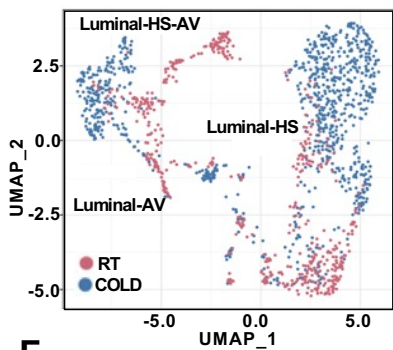

$\mathbf{F}$

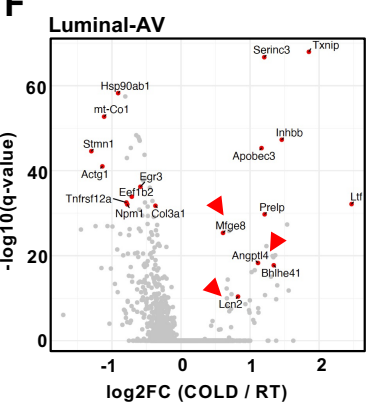

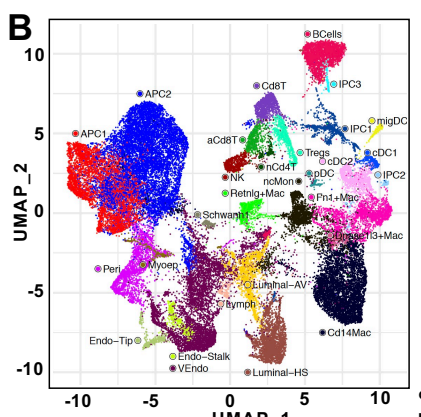
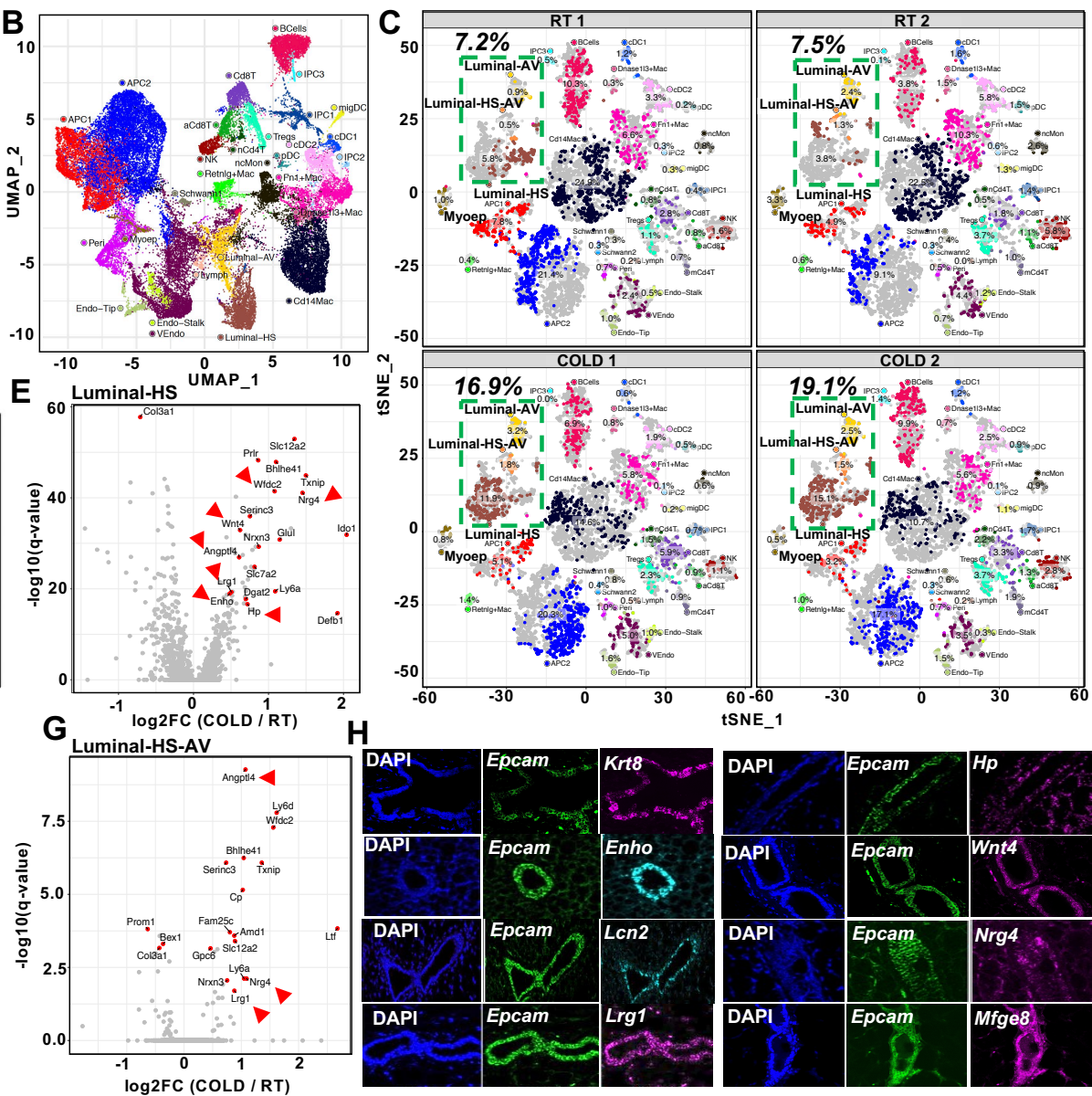

817 Figure 1. Deconstruction of mgWAT shows cold-induced remodeling of mammary epithelium

819 (A) Cartoon depiction of the scRNA-seq workflow showing isolation of stromal vascular fraction

820 (stromal cells) from mammary fat pad (mgWAT) of 10-week-old $24 \mathrm{~h}$ RT or COLD exposed

821 female mice. (B) UMAP plots of integrated single cell data from this study and 8 external datasets

822 (see Methods). Each point represents a single cell and clusters are colored by cell type. (C) t-SNE

823 plot of single cells from mammary gland and surrounding SVF colored by cell type and separated

824 by sample. Relative fractions of each cell type in each sample are indicated on each cluster. Room 
826 plot of luminal epithelial cell types from RT or cold mgWATs. (E-G) Differentially expressed 827 genes between COLD treated mice and RT animals across Luminal-HS (E), Luminal-AV (F), and

828 Luminal-HS-AV (G). Select significant DEGs (adjusted p-value $<0.05$ ) are highlighted with the 829 average log fold change between 4 degree and RT indicated on the y-axis. Genes indicated by red 830 arrows encode for secreted factors. (H) RNAScope FISH (see Materials and methods) of indicated 831 probes from mgWAT of $24 \mathrm{hr}$ cold exposed mice.

832

833 Abbreviations: APC, adipose precursor cells; IPC, immune precursor cells; Mac, macrophages; 834 ncMon, non-classical monocytes; cDC, conventional dendritic cells; migDC, migratory dendritic 835 cells; pDC, plasmacytoid dendritic cells; Tregs, regulatory T cells; mCd4T, memory Cd4 T cells; 836 nCd4T, naïve Cd4 T cells; aCd8T, activated Cd8 T cells; Myoep, myoepithelial cells; VEndo, 837 vascular endothelial cells; Endo-Tip, endothelial tip cells; Endo-Stalk, endothelial stalk cells; 838 Lymph, lymphatic endothelial cells; Peri, pericytes; Luminal-HS, hormone-sensing luminal cells; 839 Luminal-AV, secretory alveolar luminal cells; Luminal-HS-AV. 
Main Figure 2

A

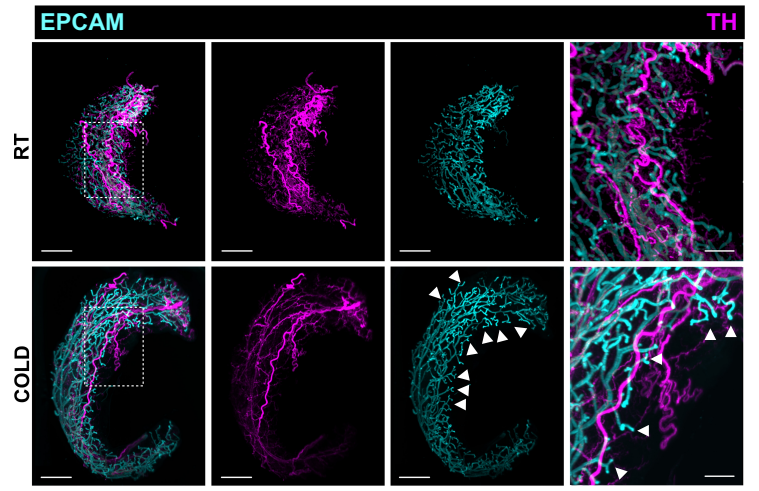

C EPCAM

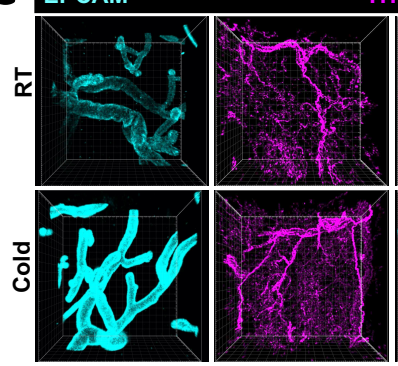

TH Neuro-ductal points D

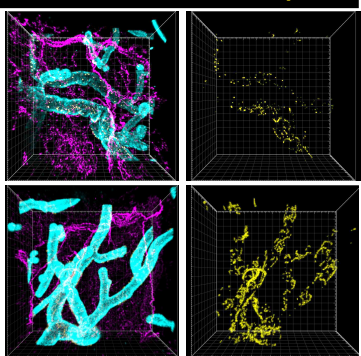

B
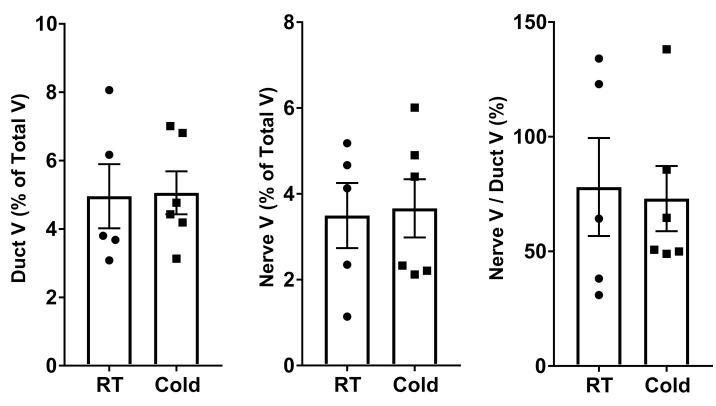

$E$

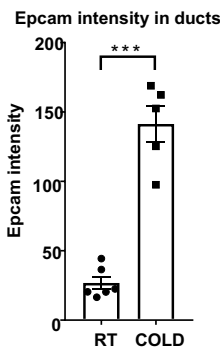

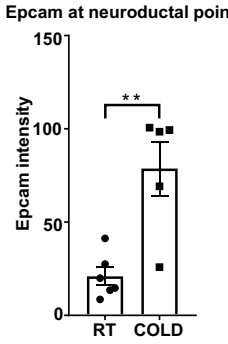

$\mathbf{F}$

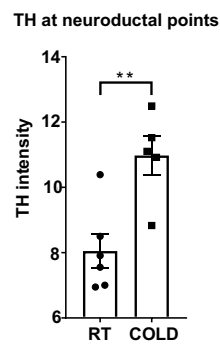

853 Figure 2. SNS fibers directly innervate mammary ductal epithelium

854 (A) Light sheet microscopy fluorescence (LSFM) images of mgWAT isolated from female mice 855 exposed to RT or COLD for $24 \mathrm{hr}$ and stained with $\mathrm{TH}$ antibody (SNS fibers) and EPCAM 856 antibody (ductal cells). Representative mgWAT images from 5-6 mice per condition. White arrows

857 show terminal ductal bifurcations under COLD condition (B) Quantification of LSFM images for 858 ductal volume (Duct V) and nerve volume (Nerve V) as a percentage of total volume (Total V), 859 and ratio of Nerve $\mathrm{V}$ and Duct V in RT or COLD mgWATs $\mathrm{N}=5-6$ per condition. (C) Confocal 860 images of mgWAT isolated from female mice exposed to RT or COLD (24 hr) and stained for

861 EPCAM and TH antibodies. Merged stainings of EPCAM and TH represent neuroductal points.

862 Representative image of 5-6 mice per condition. 
863 (D-F) Quantification of EPCAM intensity in ducts (D), EPCAM intensity at neuroductal points

864 (E), and TH intensity at neuroductal points (F). N=5-6 per condition. **, $p<0.01 ;{ }^{* * *}, \mathrm{p}<0.001$.

865

866

867

868

869

870

871

872

873

874

875

876

877

878

879

880

881

882

883

884

885 


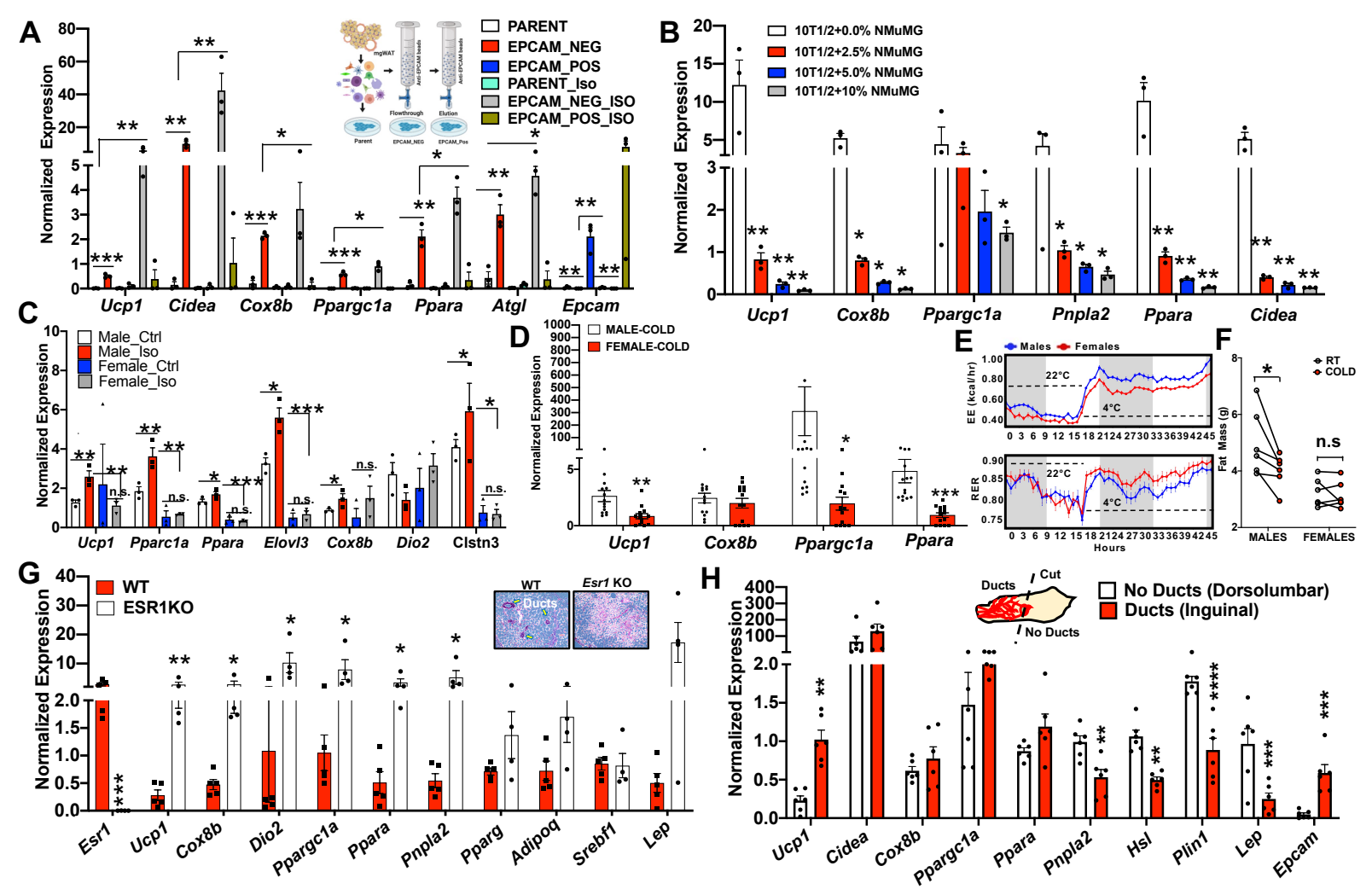

889 Figure 3. Mammary ductal cells directly inhibit adipocyte thermogenesis.

890 A) Real-time qPCR of indicated genes from beige differentiation of primary mgWAT SVF 891 (Parent), EPCAM-ve (EPCAM-NEG), and EPCAM+ (EPCAM-POS) cells treated with and 892 without $10 \mu \mathrm{M}$ isoproterenol (ISO) for $24 \mathrm{hr}$. Results are from three independent experiments. *, $893 \mathrm{p}<0.05 ; * *, \mathrm{p}<0.01 ; * * *, \mathrm{p}<0.001$. Inset represent a cartoon depiction of selecting and plating 894 EPCAM+ cells from SVFs derived from mgWATs. B) Real-time qPCR of indicated genes from 895 beige differentiated and Iso treated 10T1/2 and 2.5-10\% NMuMG mixture cells. Results are from 896 three independent experiments. *, $\mathrm{p}<0.05 ;{ }^{* *}, \mathrm{p}<0.01$. C) Real-time qPCR of indicated genes from 897 beige differentiated SVFs isolated from male and female iWATs treated with and without Iso. 898 Results are from three independent experiments. ${ }^{*}, \mathrm{p}<0.05, * *, \mathrm{p}<0.01 ; * * *, \mathrm{p}<0.001$. D) Real- 
899 time qPCR of indicated genes from $24 \mathrm{~h}$ cold exposed male and female iWATs. N=13,13 *, $\left.900 \mathrm{p}<0.05,{ }^{* *}, \mathrm{p}<0.01 ;{ }^{* * *}, \mathrm{p}<0.001 . \mathbf{E}\right)$ Energy expenditure $(\mathrm{kcal} / \mathrm{hr})$ and respiratory exchange ratio 901 (RER) of male and female mice exposed to $22^{\circ} \mathrm{C}(21 \mathrm{hr})$ and $4^{\circ} \mathrm{C}(24 \mathrm{hr})$ analyzed in Sable 902 Promethion metabolic chambers $(12 \mathrm{hr}$ light/dark cycle, $45 \mathrm{hr}$ total duration, white bar represent 903 light cycle and grey bar represent night cycle). $\mathrm{N}=6,6 . \mathrm{F})$ Fat mass of mice from (E) at RT $\left(22^{\circ} \mathrm{C}\right)$ 904 and $\operatorname{COLD}\left(4^{\circ} \mathrm{C}\right) . \mathrm{N}=6,6 .{ }^{*}, \mathrm{p}<0.05$; n.s, not significant. G) Real-time qPCR of indicated genes 905 from $24 \mathrm{hr}$ cold exposed WT and Esr $1 \mathrm{KO}$ mgWATs. Inset picture shows histological section of 906 mgWATs from WT and Esr1 KO mice. Arrows are pointing towards ducts in WT. N=5,5 *, $\left.907 \mathrm{p}<0.05,{ }^{* *}, \mathrm{p}<0.01 . \mathrm{H}\right)$ Real-time qPCR of indicated genes from dorsolumbar or inguinal parts of 908 cold-exposed 5-week-old mice. Inset picture shows cartoon depiction of ducts (inguinal) and no 909 ducts (dorsolumbar). Dotted line represents cut site to separate inguinal and dorsolumbar regions 910 of mgWAT. N=6,6. *, p<0.05, **, $p<0.01 ; * * *, p<0.001$

911

912

913

914

915

916

917

918

919

920

921 

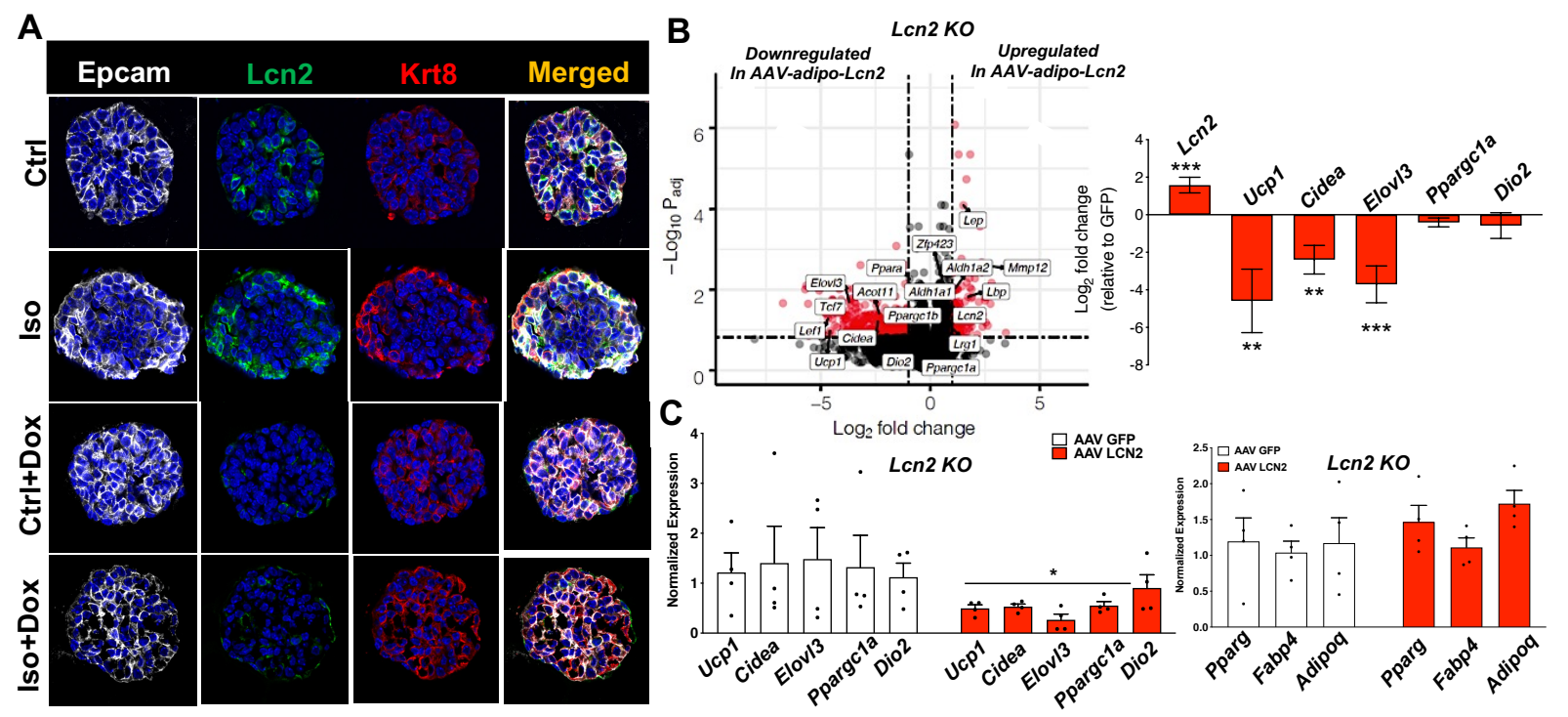

924 Figure 4. LCN2 preserves mgWAT adiposity.

925 A) Confocal images of immunostaining of indicated antibodies in the organoids derived from 926 K8rtTA-DTA mice (see Methods) treated with and without Iso and Doxycycline (Dox).

927 Representative images from 3 organoid experiments. B) Volcano plot of DEGs from the mgWAT 928 of Lcn2 $\mathrm{KO}$ mice treated with adipose-specific AAV-LCN2 or AAV-GFP and represented as a 929 fold change of LCN2/GFP ratio as a function of p-value. Genes labelled are either induced $(+)$ or 930 repressed (-) by Lcn $2 . * *, p<0.01 ; * * *, p<0.001 . \mathrm{N}=4,4$. C) Real-time qPCR of indicated genes 931 from the mgWATs of LCN2KO or WT mice treated with AAV-LCN2 or AAV-GFP. N=4,4. *, $932 \mathrm{p}<0.05$ 
Extended Data Figure 1

A

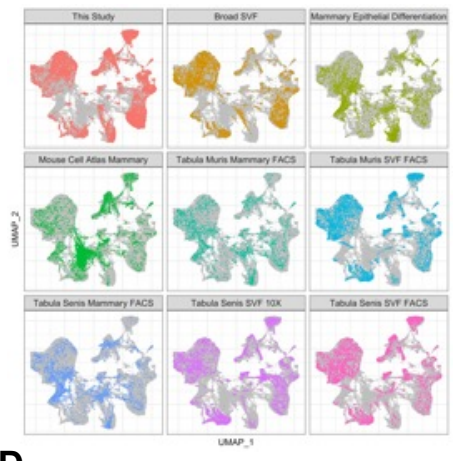

D

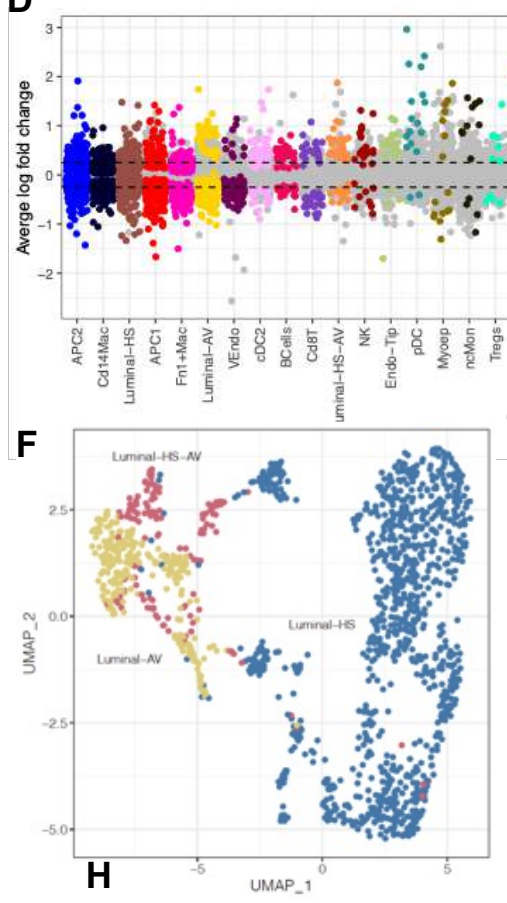

B

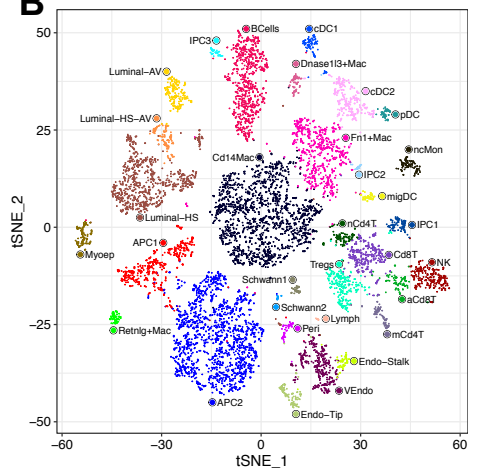

C

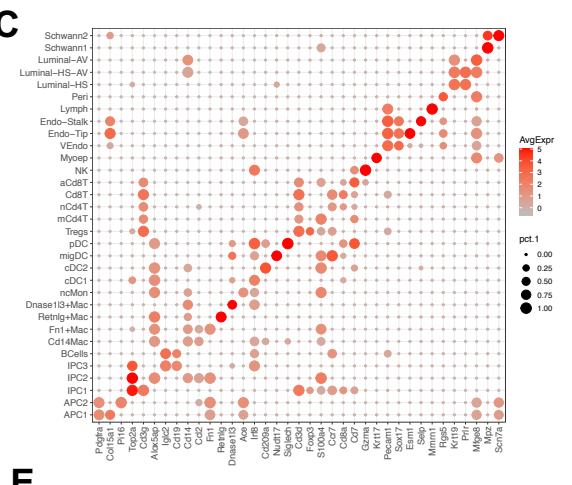

E

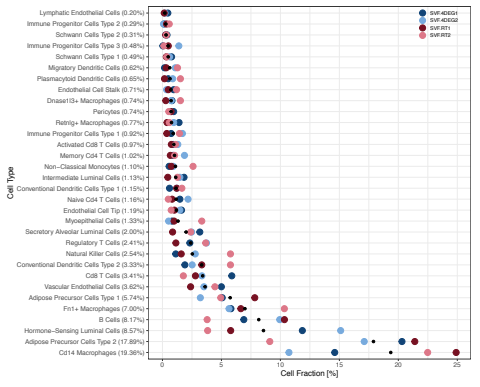

G
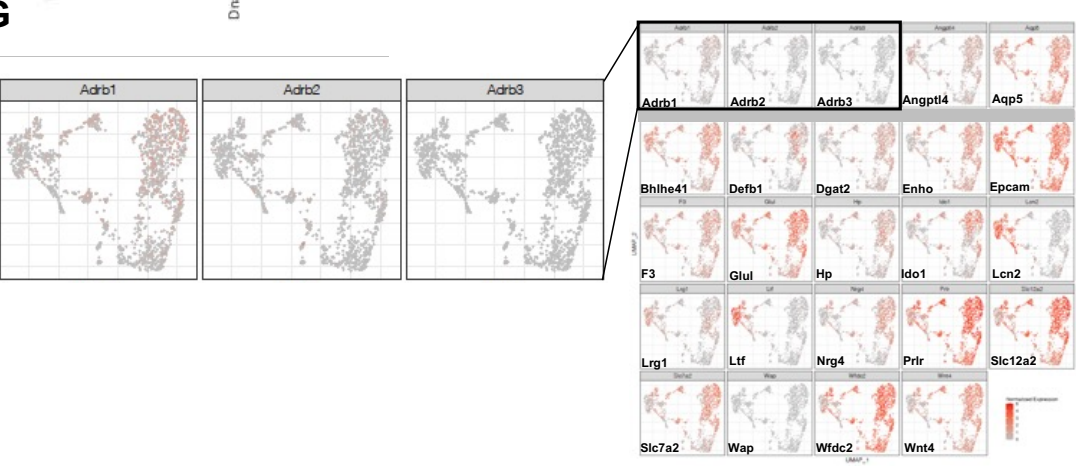

Mature iWAT

Adipocytes

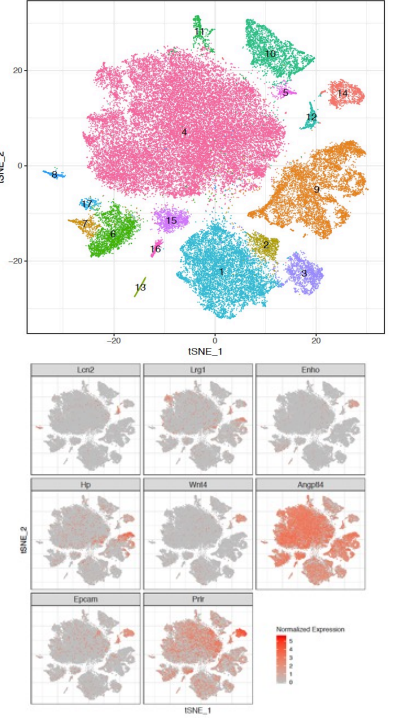

Male iWAT SVF
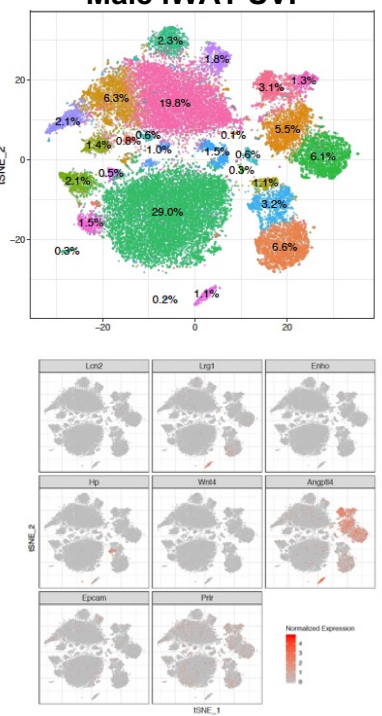

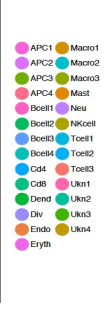

Female mgWAT SVF
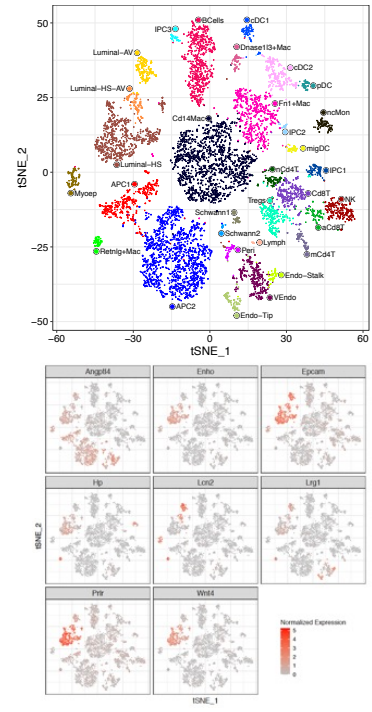
938 Figure 1. Cold-associated increase in cell percentages of luminal epithelium subtypes.

939 A) UMAP plots of integrated single cell data from this study and 8 external datasets (see Methods

940 and Table 1). Each point represents a single cell and are colored by dataset. B) t-SNE plot of single

941 cells from mammary gland and surrounding SVF from this study colored by cell type. Cell types

942 were identified based on expression of canonical marker genes. C) Expression of known canonical

943 markers for cell types in the SVF and mammary gland. Color corresponds to average expression

944 level and size corresponds to percentage of cells which express the gene within the cluster. D)

945 Differentially expressed genes between COLD (24 hr) treated mice and RT animals across all cell

946 types. Significant DEGs (adjusted p-value $<0.05$ ) are highlighted with the average log fold change

947 between 4 degree and RT indicated on the y-axis. Cell types are ordered on the x-axis based on

948 the number of significant DEGs. E) Relative fractions of cell types within each sample. Black dots

949 indicate average relative fractions across all samples. F) Aggregated UMAP plot of subclustering

950 of luminal single cells from RT and cold-exposed mice. G) UMAP plots of normalized gene

951 expression levels for genes of interest in luminal cells. H) tSNE plots of cell type clusters and

952 normalized gene expression levels for genes of interest across multiple datasets including: female

953 mgWAT SVFs, male iWAT SVFs and mature mgWAT adipocytes.

954

955

956

957

958

959

960 
A

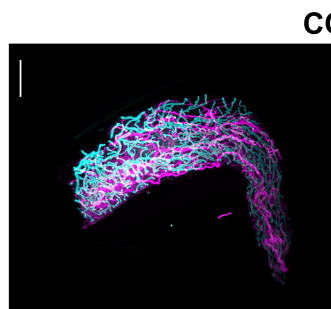

COLD
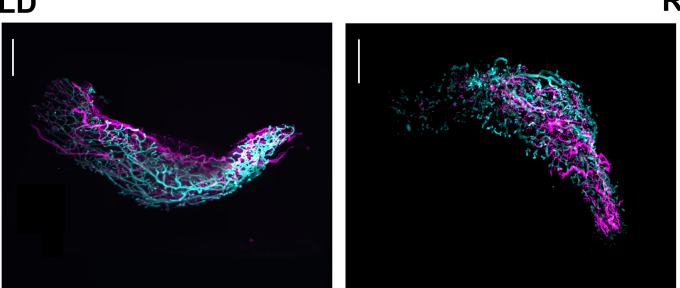

RT
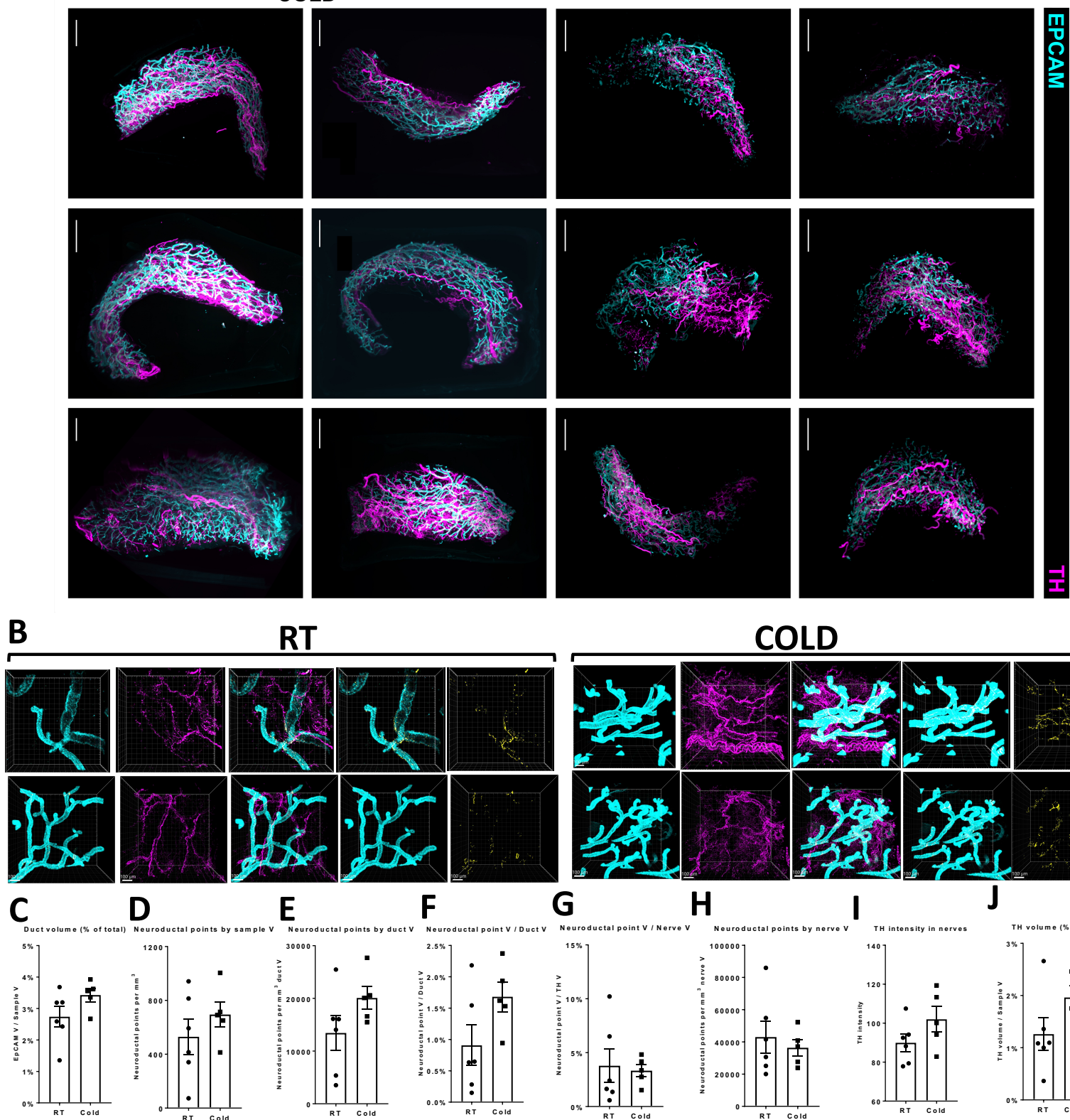

D

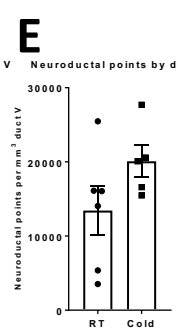

$F$

G

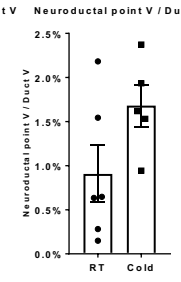

COLD

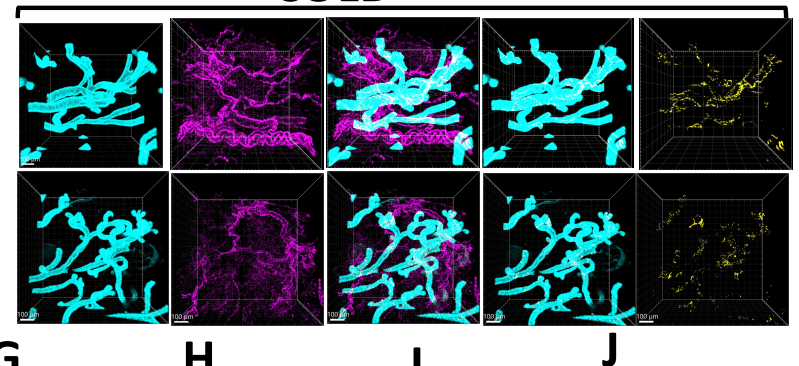

H

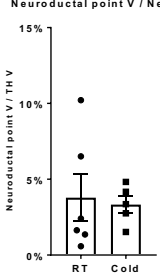

I

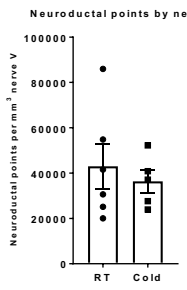

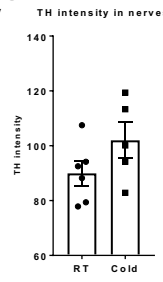

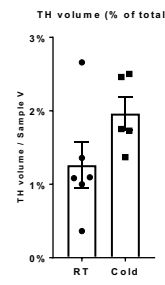

963 Figure 2. SNS fibers directly innervate ductal epithelial cells.

964 A) Light sheet microscopy fluorescence (LSFM) images of mgWAT isolated from female mice

965 exposed to RT or COLD for $24 \mathrm{hr}$ and stained with $\mathrm{TH}$ antibody (SNS fibers) and EPCAM

966 antibody (ductal cells). $\mathrm{N}=6,6$. B) Confocal images of mgWAT isolated from female mice exposed 
967 to RT or COLD for 24hr and stained for EPCAM and TH antibodies. Merged staining of EPCAM

968 and TH represent neuroductal points. Representative images of 5-6 mice per condition. C-J)

969 Quantification of indicated parameters of images from (B). N=5-6 mice per condition.

970

971

972

973

974

975

976

977

978

979

980

981

982

983

984

985

986

987

988

989 
Extended Data Figure 3
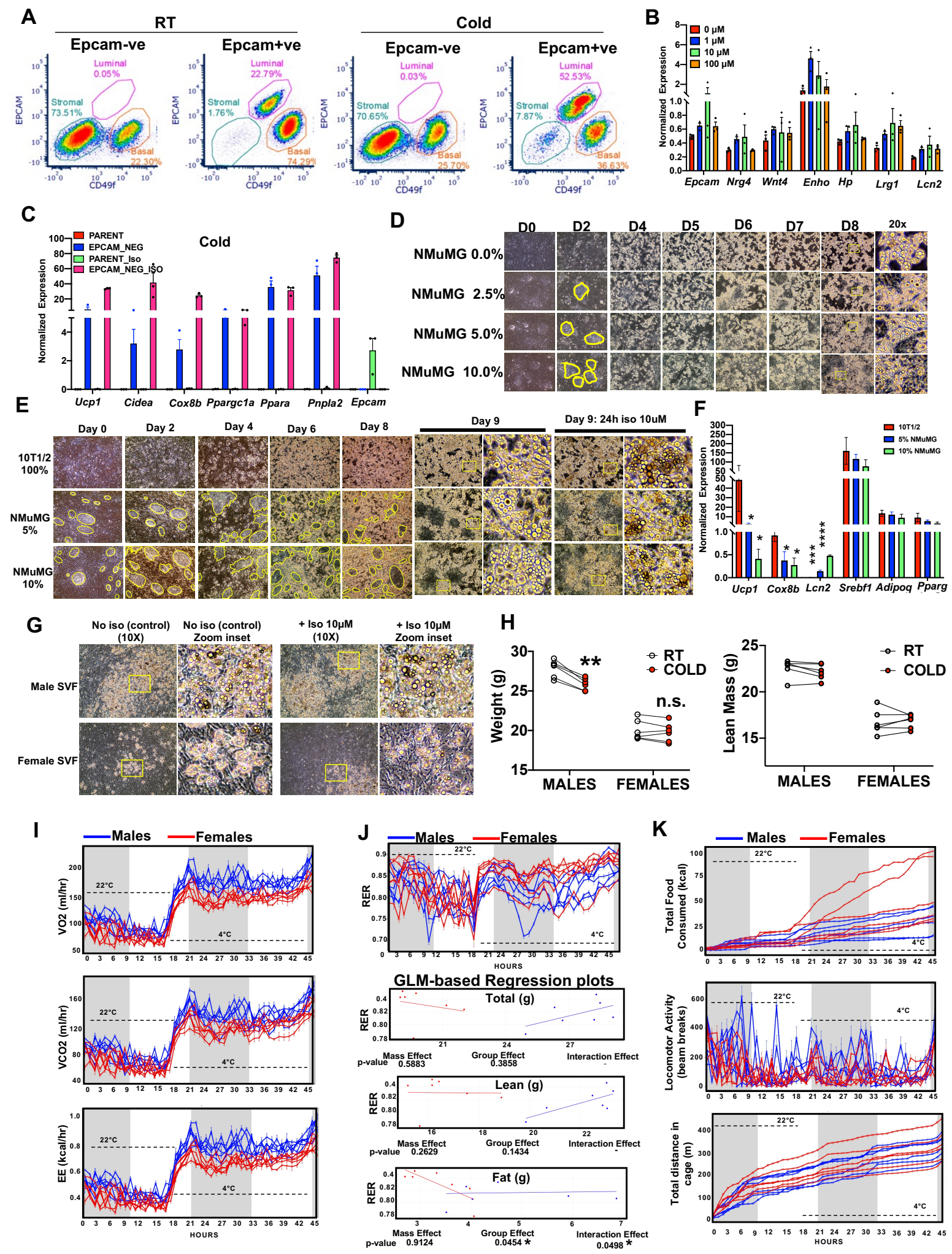


\section{Figure 3. Mammary gland epithelium inhibits cold-induced adipocyte thermogensis}

993 A) Representative FACS plot of CD49f and EPCAM expression in EPCAM bead selected 994 EPCAM+ or EPCAM-ve epithelial cells from RT or cold mice. 2 mice per condition and 4 995 mammary fat pads per mouse. Representative data from 4 independent experiments. B) Real-time 996 qPCR of indicated genes in beige differentiated SVFs from mgWAT treated with indicated

997 concentration of Iso for $5 \mathrm{hr}$. C) Real-time qPCR of indicated genes from beige differentiated 998 primary mgWAT SVF (Parent) and EPCAM-ve (EPCAM-NEG) cells isolated from cold exposed 999 mice treated with and without $10 \mu \mathrm{M}$ isoproterenol (ISO) for $24 \mathrm{hr}$ ex vivo. Results are from three 1000 independent experiments. D) Images showing cell morphology of D0-D8 beige differentiated 1001 10T1/2 and NMuMG (0, 5, and 10\%) mixture cells. Yellow enclosures show NMuMG epithelial 1002 cells surrounded by $10 \mathrm{~T} 1 / 2$ cells. E) Images showing cell morphology of D0-D9 beige 1003 differentiated 10T1/2 and NMuMG (0-10\%) mixture cells. Yellow enclosures show NMuMG 1004 epithelial cells surrounded by 10T1/2 cells. F) Real-time qPCR of indicated genes from cells in 1005 (E). Results are from three independent experiments. *, $<<0.05, * * *, p<0.001$. G) Images showing 1006 cell morphology of beige differentiated SVFs isolated from male and female iWATs. H) Body 1007 weight and lean mass of male and female mice before (RT) and after $24 \mathrm{hr}$ cold exposure (COLD). $1008 \mathrm{~N}=6,6 . * *, \mathrm{p}<0.01$. I-K) Individual mice data for oxygen consumption (VO2 $\mathrm{ml} / \mathrm{hr})$, carbon 1009 dioxide production (VCO2 ml/hr), and energy expenditure (EE kcal/hr) (I), respiratory exchange 1010 ratio (RER) and generalized linear model (GLM)-based regression plots of RER with total body 1011 weight (Total), lean mass (Lean) and fat mass (Fat) as co-variates (ANCOVA) showing p-value 1012 for Mass effect, Group effect, and Interaction effect. *, $<<0.05(\mathrm{~J})$, and total food consumed (kcal), 1013 locomotor activity (beam breaks), and total distance in cage $(\mathrm{m})(\mathrm{K})$ of male and female mice 1014 exposed to $22^{\circ} \mathrm{C}$ for $21 \mathrm{hr}$ and $4^{\circ} \mathrm{C}$ for $24 \mathrm{hr}$ in Sable Promethion metabolic chambers $(12 \mathrm{hr}$ 
1015 light/dark cycle, $45 \mathrm{hr}$ total duration, white bar represents light cycle and grey bar represents night 1016 cycle). $\mathrm{N}=6,6$.

1017

1018

1019

1020

1021

1022

1023

1024

1025

1026

1027

1028

1029

1030

1031

1032

1033

1034

1035

1036

1037 


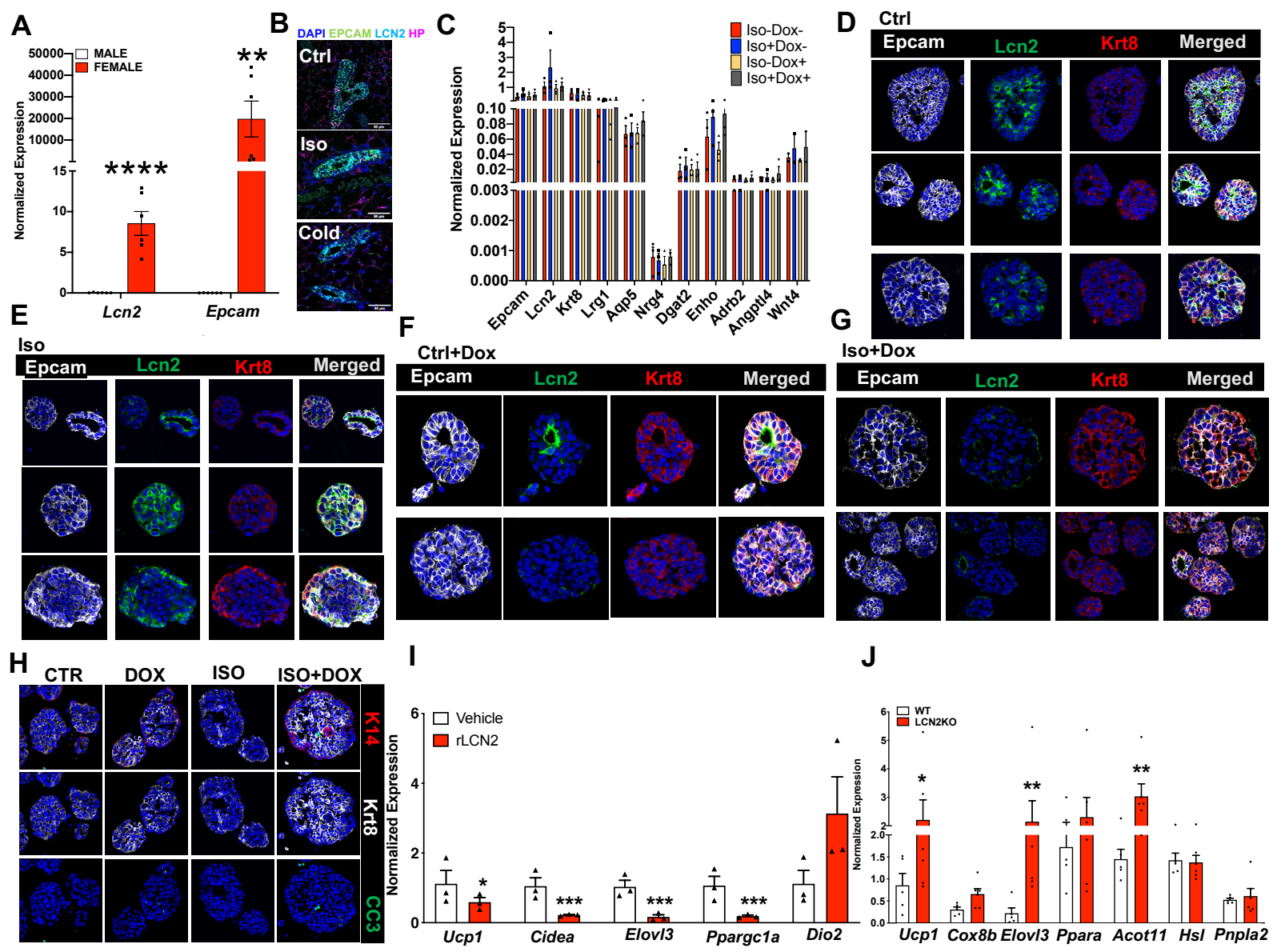

1040 Figure 4. LCN2 is a cold-induced mammokine involved in blocking cold-induced adipocyte

1041 UCP1 expression

1042 A) Real-time qPCR of indicated genes from $24 \mathrm{hr}$ cold-exposed male and female iWATs. **, $1043 \mathrm{p}<0.01 ; * * *, \mathrm{p}<0.001$. B) RNAScope FISH (see Materials and Methods) of indicated probes from 1044 mgWAT of RT or $24 \mathrm{hr}$ isoproterenol or cold-exposed mice. C) Real-time qPCR of indicated genes 1045 in organoids derived from K8rtTA-DTA mice treated with and without ISO and Doxycycline 1046 (Dox). Results from 3 organoid experiments. D-H) Confocal images of immunostaining of 1047 indicated antibodies in organoids derived from K8rtTA-DTA mice treated with and without Iso 1048 and Dox. Representative images from 2-3 organoid experiments. I) Real-time qPCR of indicated 
1049 genes from beige differentiation mgWAT SVFs derived from Lcn2 KO mice treated with and 1050 without recombinant Lcn2 (rLcn2). *, $<<0.05 ;{ }^{* * *}, \mathrm{p}<0.001$ J) Real-time qPCR of indicated genes 1051 from $24 \mathrm{hr}$ cold exposed WT and Lcn2 KO mgWATs. N=5,5. *, $<<0.05 ;{ }^{* *}, \mathrm{p}<0.01$ 1052

1053 1054 1055 1056 1057 1058 1059 1060 1061 1062 1063 1064 1065 1066 1067 


\section{Supplementary Files}

This is a list of supplementary files associated with this preprint. Click to download.

- SantosNatureMovie1.pptx

- SantosNatureMovie2.pptx

- SupplementaryTable1.xlsx 